\title{
Classical limit of the Quantized Hyperbolic Toral Automorphisms
}

\author{
Mirko Degli Esposti, Sandro Graffi, Stefano Isola \\ Dipartimento di Matematica, Università di Bologna, I-40127 Bologna, Italy \\ Received: 15 September 1993/in revised form: 10 February 1994
}

\begin{abstract}
The canonical quantization of any hyperbolic symplectomorphism $A$ of the 2-torus yields a periodic unitary operator on a $N$-dimensional Hilbert space, $N=\frac{1}{h}$. We prove that this quantum system becomes ergodic and mixing at the classical limit $(N \rightarrow \infty, N$ prime) which can be interchanged with the time-average limit. The recovery of the stochastic behaviour out of a periodic one is based on the same mechanism under which the uniform distribution of the classical periodic orbits reproduces the Lebesgue measure: the Wigner functions of the eigenstates, supported on the classical periodic orbits, are indeed proved to become uniformly spread in phase space.
\end{abstract}

\section{Contents}

1. Von Neumann definition of the quantum ergodicity and mixing properties. Statement of the main results. ..........................

2. Koopman operator on invariant lattices and periodic orbits. Limits of atomic invariant measures supported on periodic orbits via Kloosterman

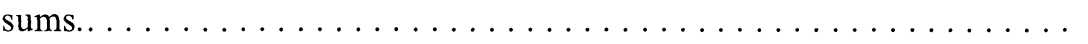

3. Quantization of toral automorphisms. Discrete Wigner functions. Support on classical periodic orbits, relation with the Koopman operator and explicit construction of the quantum eigenvectors..............

4. Classical limit of the matrix elements of the observables via Weil-Deligne exponential sums. Weak-* convergence of the Wigner functions. Proof of the main results. ......................... 495

Appendix A. Some basic results out of number theory. . . . . . . . 503

\section{Introduction}

The quantization of any linear hyperbolic symplectomorphism $A$ of the 2-torus $\mathbb{T}^{2}$ yields a unitary operator $V_{A}$ acting on a Hilbert space of finite dimension $N=h^{-1}$, in agreement with the well known physical intuition that a compact phase space 
allows only for a finite number of quantum states. Moreover, $V_{A}$ is periodic, i.e. there is $p=p(N) \in \mathbb{N}$ such that $V_{A}^{p}=I_{d}$ (up to a phase). Since the linear hyperbolic symplectomorphisms of the 2-torus represent the best known examples of "chaotic" systems [AA], this periodicity makes the classical limit $N \rightarrow \infty$, in which the stochastic properties should be recovered, an apparently non-trivial one (see, e.g., [FMR]).

The purpose of this paper is to take on the problem for the two most basic stochastic properties, namely ergodicity and mixing, and to prove that, at least on subsequences $\left\{N_{k}\right\}$ of prime numbers, they can be actually recovered in the strongest possible sense, namely: the "classical limit" $N_{k} \rightarrow \infty$ can be interchanged with the "time-average" limit $m \rightarrow \infty$. The mechanism underlying this recovery is exactly the same one generating the intrinsic, i.e., maximal entropy ergodic invariant measure of the symplectomorphism, out of the uniform spreading over the torus of periodic orbits of increasing period. This can be directly transported to the quantum case via support and invariance properties of the Wigner functions corresponding to the eigenstates of $V_{A}$. Concerning the above table of contents, we remark:

Sect. 1: A difficulty in the quantum ergodicity notion of von Neumann [VN] (which on the other hand trivially holds whenever the propagator spectrum is pure-point and simple) lies in its non-obvious reducibility to the classical definition (see e.g. [E2]), which is instead here explicitly verified.

Sect. 2: The weak-* convergence to the Lebesgue measure $d \mu$ on $\mathbb{T}^{2}$ of the averaged atomic measures concentrated on all periodic points of any hyperbolic $A \in \mathrm{SL}(2, \mathbb{Z})$ is a classical result of Bowen and Sinai (see e.g. [M,PP]). For the particular case under examination, by reducing the integrals over the periodic orbits to Kloosterman sums (see e.g. [Ka1]) we prove a stronger result: namely, the weak-* convergence $d \mu_{\gamma} \rightarrow d \mu$, where $d \mu$, is the invariant measure supported on any single periodic orbit sequence $\left\{\gamma_{N}\right\}$ belonging to the invariant lattice of all points of rational coordinates with common denominator $N$, and $N \rightarrow \infty$ over splitting primes relative to $A$. We also determine the spectral properties of the Koopman operator restricted to the $L^{2}$ spaces of the invariant atomic measures, concentrated on the sublattices of $\mathbb{T}^{2}$ generated by the points of rational coordinates.

Sect. 3: The first quantization of a subclass of matrices in $\operatorname{SL}(2, \mathbb{Z})$ in a $N$-dimensional Hilbert space, $N=h^{-1}$, was obtained by Berry and Hannay $[\mathrm{BH}]$; the canonical quantization of the observables valid for any $A \in \operatorname{SL}(2, \mathbb{Z})$ has been obtained in [DE]. The spectral, periodicity and arithmetic properties of the quantum propagator $V_{A}$ are studied in [BV, E1, Ke1, $\left.\mathrm{Ke} 2, \mathrm{Ke} 3, \mathrm{PV}\right]$ in addition to $[\mathrm{BH}, \mathrm{DE}]$; if representations and classical limits are ignored, and the torus is instead the configuration space, the corresponding quantum system can be studied as a pure Weyl algebra automorphism also for $h$ irrational [BNS, N]. We employ here the canonical quantization procedure of [DE], based on the representations of the Heisenberg group $\mathbb{H}_{n}\left(\mathbb{Z}^{n}\right)$, in complete analogy with the well known construction of the Schrödinger representation out of the Heisenberg group $\mathbb{H}_{n}\left(\mathbb{R}^{n}\right)$ (see $[\mathrm{F}])$. The discrete Wigner transform has also been first introduced in $[\mathrm{BH}]$, essentially as a discretization of the standard one defined on $\mathbb{R}^{2}$, and its most relevant properties, including the support on the classical periodic orbits, have been studied also in [BV, E1, Ke1, Ke2, Kn, PV, LV]. Here the discrete Wigner transform is directly defined out of the representations of $\mathrm{IH}_{n}\left(\mathbb{Z}^{2}\right)$; in particular, this 
makes possible to remove the restriction to $N$ even of $[\mathrm{BH}]$ as well as to determine the normalization factor. The invariance of the Wigner functions of the eigenvectors of the quantum propagator allows us to relate its spectral properties to those of the Koopman operator generated by the classical dynamical system. Moreover, a simplified version is presented of the construction (obtained in [E1, DE]) of the (even) eigenvectors of the quantum propagator in terms of the evolution under $A$ of well determined subsets ("straight lines" or "Lagrangian manifolds" and "ideal straight lines") of classical periodic points. This construction yields a representation of the Wigner functions which illuminates the suppression of its sign alternance at the classical limit.

Sect. 4: The proof of the results rests on the convergence of the diagonal matrix elements of the quantum observables to the ergodic mean of the corresponding classical symbols at the classical limit. Results of this type, at least on subsequences, have been proved in [S, CdV, HMR, Z] in the context of geodesic flows on compact manifolds with negative curvature and of ergodic flows on constant energy surfaces, respectively. Here we give two independent convergence proofs. The first is based on a direct computation of the matrix elements in terms of exponential sums fulfilling the Weil-Deligne estimates (see e.g. [De1, De2, Sc]); the second is based on the representation of the matrix elements as Fourier coefficients of the Wigner functions, which are shown to tend to a constant limit. The intuition behind this result is that, since the Wigner functions are supported on classical periodic orbits, their weak-* convergence to the Lebesgue measure follows from the uniform distribution of all classical periodic orbits in phase space.

Appendix A. We collect here, for convenience of exposition, the basic definition and results on Gaussian sums, quadratic residues, and exponential sums of rational functions (in particular, Kloosterman sums) needed for the proofs of Sects. 4, 5 .

\section{Basic Definitions, Notation and Statement of the Results}

In analogy to the standard concept of classical discrete dynamical system, i.e. the triple $(M, T, \mu)$, where $T$ is an automorphism of the measure space $M$ leaving the measure $\mu$ invariant, the triple $(\mathscr{H}, \mathscr{A}, V)$, where $\mathscr{H}$ is a separable Hilbert space, $V$ : $\mathscr{H} \leftrightarrow \mathscr{H}$ a unitary bijection in $\mathscr{H}$ with $\sigma_{\text {css }}(V)=\emptyset$, and $\mathscr{A}$ the algebra of the observables in $\mathscr{H}$ will be referred to as a quantum discrete dynamical system. Then the definition of quantum ergodicity of von Neumann [VN] in the present case reads:

Definition 1 (Ergodicity). The quantum discrete dynamical system $(\mathscr{H}, \mathscr{A}, V)$ is ergodic iff, for any observable $\hat{f} \in \mathscr{A}$ :

$$
\lim _{m \rightarrow \infty} \frac{1}{m} \sum_{k=0}^{m-1}\left\langle\psi, V^{k} \hat{f} V^{-k} \psi\right\rangle=\sum_{n=0}^{\infty}\left|a_{n}\right|^{2}\left\langle e_{n}, \hat{f e_{n}}\right\rangle,
$$

where $\psi=\sum_{n=0}^{\infty} a_{n} e_{n}$ is any vector in $\mathscr{H}$ expanded on the eigenstates $\left\{e_{n}\right\}$ of $V$.

Setting $\hat{f}(k)=V^{k} \hat{f} V^{-k}$ the consequent definition of mixing is then given by: 
Definition 2 (Mixing). The quantum dynamical system ( $\mathscr{H}, \mathscr{A}, V)$ is mixing iff, for any pair of observables $\hat{f} \hat{g}$ :

$$
\begin{gathered}
\lim _{l \rightarrow \infty} \lim _{m \rightarrow \infty} \frac{1}{m} \sum_{k=0}^{m-1}\left\langle\psi, V^{l} \hat{f}(k) V^{-l} \hat{g}(k) \psi\right\rangle \\
=\left(\lim _{m \rightarrow \infty} \frac{1}{m} \sum_{k=0}^{m-1}\langle\psi, \hat{f}(k) \psi\rangle\right)\left(\lim _{m \rightarrow \infty} \frac{1}{m} \sum_{k=0}^{m-1}\langle\psi, \hat{g}(k) \psi\rangle\right) .
\end{gathered}
$$

Remark. It is a well known simple fact that (1.1) holds provided either the eigenvalues of $V$ are simple, or the matrix elements $\left\langle e_{r}, \hat{f e_{s}}\right\rangle$ among different eigenvectors corresponding to the same eigenvalues vanish identically. Even in this case, however, the "time average" depends on $\left|a_{s}\right|: s=1, \ldots$, and hence on the initial state $\psi$. By the normalization condition, the dependence on the initial state disappears if for instance $\left\langle e_{s}, \hat{f e_{s}}\right\rangle$ is independent of $s$. This property does not hold in general, but only at the classical limit, provided one deals with the quantization of a classically ergodic system as verified in [CdV, HMR, S, Z]. Moreover under the assumption $\sigma_{\text {ess }}=\emptyset$ the quantum system is obviously quasi-periodic, which prevents the validity of the mixing property. This definition becomes interesting only at the classical limit.

To state the results of the present paper, we assume from now on:

(1) Classical dynamical system $(M, T, \mu)$ : let $M$ be the 2 -torus $\mathbb{T}^{2}=\mathbb{R}^{2} / \mathbb{Z}^{2}$ (points on $\mathbb{T}^{2}$ are denoted by $\left.x=(p, q) \in[0,1] \times[0,1]\right) ; \mu$ the Lebesgue measure on $\mathbb{T}^{2}$; $T$ the hyperbolic automorphism of $\mathbb{T}^{2}$ generated by the matrix

$$
A=\left(\begin{array}{ll}
a & b \\
c & d
\end{array}\right)
$$

such that $A \in \mathrm{SL}(2, \mathbb{Z})$, i.e., $(a, b, c, d) \in \mathbb{Z}, a d-b c=1$ and $|a+d|>2$.

Via periodicity and Fourier expansion, $A$ acts on points $x=(q, p)$ and on suitably smooth functions $f(x)$ on $M$ respectively as:

$$
\left\{\begin{array}{l}
A x=((a q+b p) \quad \bmod 1,(c q+d p) \quad \bmod 1 ; \\
f(A x)=\sum_{n \in \mathbb{Z}^{2}} f_{n} e^{2 \pi i\left\langle A^{\prime} n, x\right\rangle}
\end{array}\right.
$$

where $A^{t}$ is the transposed matrix of $A$. $\mu$ is invariant because $\operatorname{det}(A)=1$;

(2) The quantum dynamical system $\left(\mathscr{H}, \mathscr{A}, V_{A}\right)$ is the canonical quantization of the former one. Namely (details in Sect. 3 below), with $h=\frac{1}{N}, \mathscr{H} \equiv \mathscr{H}_{N}=L^{2}\left(S^{1}, \mu_{N}\right)$, where $\mu_{N}$ is the atomic measure on $S^{1}$ given by $\left(e^{2 \pi i q} \in S^{1}\right)$ :

$$
\mu_{N}(q):=\frac{1}{N} \sum_{l=0}^{N-1} \delta\left(q-\frac{l}{N}\right) .
$$

The Hilbert space $\mathscr{H}_{N}=L^{2}\left(S^{1}, \mu_{N}\right)$ is $N$-dimensional; the vectors $|k\rangle=\delta_{k / N}^{q}$ for $k \in\{0,1, \ldots, N-1\} \equiv \mathbb{Z}_{N}=\mathbb{Z} / N \mathbb{Z}$ are a basis of $\mathscr{H}_{N}$, and the inner product between $f, g \in \mathscr{H}_{N}$ is given by:

$$
\langle f, g\rangle:=\frac{1}{N} \sum_{l=0}^{N-1} \bar{f}\left(\frac{l}{N}\right) g\left(\frac{l}{N}\right) .
$$


The algebra $\mathscr{A}$ is the $*$-algebra of the observables on $\mathscr{H}_{N}$ generated by the canonical quantization: let $A\left(\mathbb{T}^{2}\right)$ be the Banach space of all functions $f: \mathbb{T}^{2} \rightarrow \mathbb{C}$ such that

$$
\|f\|_{A}:=\sum_{n \in \mathbb{Z}^{2}}\left|f_{n}\right|<+\infty .
$$

Then, if $\hat{T}(n): n \in \mathbb{Z}^{2}$ is the canonical quantization of the basic observables

$$
T(n)=\exp (2 \pi i\langle n, x\rangle)=\exp \left[2 \pi i\left(n_{1} q+n_{2} p\right)\right],
$$

the quantization of any $f \in A\left(\mathbb{T}^{2}\right)$ is

$$
\hat{f}=\sum_{n \in \mathbb{Z}^{2}} f_{n} \hat{T}(n),
$$

and (construction recalled in Sect. 3.3)

$$
W(\phi, \psi)(m)=\sum_{k=0}^{N-1} e^{-\frac{2 \pi \pi 2 k m_{2}}{N}} \bar{\phi}\left(m_{1}+k\right) \psi\left(m_{1}-k\right): m \in \mathbb{Z}_{N}^{2}
$$

is the (discrete) Wigner transform of the vectors $(\phi, \psi) \in \mathscr{H}_{N}$.

(3) The unitary bijection $V_{A}$ is the "quantum propagator," i.e. the quantization (construction recalled in Sect. 3.1) of the action of the symplectomorphism $A$ on the "classical observables": this means that, if $f \mapsto \hat{f}$ under canonical quantization, then $f(A x) \mapsto V_{A} \hat{f} V_{A}^{-1}$. Therefore the quantum discrete dynamics of any observable $\hat{f}$ is defined as

$$
\hat{f} \mapsto V_{A}^{k} \hat{f} V_{A}^{-1}: k \in \mathbb{Z} .
$$

We denote by $\exp i\left(\lambda_{n}^{(N)}\right): n=0, \ldots, N-1$ the (repeated) eigenvalues of $V_{A}$, by $e_{n}^{(N)}: n=0, \ldots, N-1$ the corresponding (orthonormal) eigenvectors and by $p=p(N)$ the period, namely the minimum $p>1$ such that $V_{A}^{p}=I_{d}$.

(4) The prime number $N$ is decomposed or splitting (for this notion see e.g. [H], Sect. 26) with respect to the characteristic polynomial of $A$, i.e. there exists $x \in \mathbb{Z}_{N}$ such that $\operatorname{Tr} A^{2}-4=x^{2} \bmod N$.

Then we have:

Main Theorem. Let $f, g \in A\left(\mathbb{T}^{2}\right)$ and let $N \in \Gamma, \Gamma$ being any increasing sequence of primes such that $\frac{N}{p(N)}<C$ for some $C$ independent of $N \in \Gamma$. Let furthermore $e_{n}^{(N)}: n=0, \ldots, N-1$ be a normalized eigenvectors basis of $V_{A}$. Then, whenever $\lambda_{r}^{(N)}=\lambda_{s}^{(N)}$ :

(1) The following integral representations hold:

$$
\begin{gathered}
\left\langle e_{r}^{(N)}, \hat{f} e_{s}^{(N)}\right\rangle=\int_{\mathbb{T}^{2}} f(x) d \Omega_{e_{e}, e_{s}}^{(N)}(x), \\
\left\langle e_{r}^{(N)}, V_{A}^{-n} \hat{f} V_{A}^{n} \hat{g} e_{s}^{(N)}\right\rangle=\int_{\mathbb{T}^{2}} f\left(A^{n} x\right) g(x) d \Omega_{e_{r}, e_{s}}^{(N)}(x)+R(f, g ; n, N),
\end{gathered}
$$

where the sequences $\{R(f, g ; n, N)\}_{N \in \Gamma} \rightarrow 0$ as $N \rightarrow \infty$ uniformly with respect to $n$ and $d \Omega_{e_{1}, e_{s}}^{(N)}(x)$ is the complex-valued measure on $\mathbb{T}^{2}$ defined as follows:

$$
d \Omega_{e_{1}, e_{s}}^{(N)}(x):=W\left(e_{r}^{(N)}, e_{s}^{(N)}\right)(p, q) d \mu_{N}(q) \times d \mu_{N}(p) .
$$


(2) $\mathrm{d} \Omega{ }_{e_{r}, e_{s}}^{(N)}(x)$ converges vaguely to the Lebesgue measure on $\mathbb{T}^{2}$ as $N \rightarrow \infty$ if $r=s$, and to the null measure if $r \neq s$.

(3) There is $D>0$ independent of $N$ such that

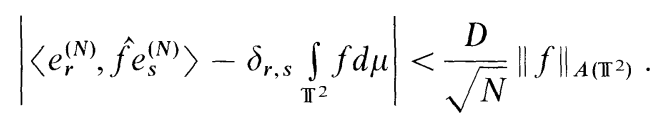

Remarks.

(1) Since $d \mu_{N}(q) \times d \mu_{N}(p)$ is obviously vaguely convergent to the Lebesgue measure as $k \rightarrow \infty$, it is enough to prove, confirming earlier numerical evidence (see e.g. $[\mathrm{LV}]$ the "equidistribution" of the Wigner functions, namely that they converge vaguely either to $1(r=s)$ or to $0(r \neq s)$.

(2) By (1.12), Assertion (2) implies

$$
\lim _{N \rightarrow \infty, N \in \Gamma}\left\langle e_{r}^{(N)}, \hat{f e_{s}^{(N)}}\right\rangle=\delta_{r, s} \int_{\mathbb{T}^{2}} f d \mu
$$

and, conversely, (1.15) implies Assertion (2). However, the two statements will be proved independently.

(3) The existence of at least a sequence $\Gamma$ fulfilling the condition of the Main Theorem (actually, the fact that almost any sequence of primes has the above property) is a consequence of the Artin conjecture, whose falsity would imply the falsity of the Generalized Riemann hypothesis (see e.g. [RM]). Detailed heuristic and numerical investigations on the behaviour of $p(N) / N$ supporting the above genericity are performed in [K2, BV].

Any easy consequence of the Main Theorem will be the following

Main Corollary. Under the same assumptions of the Main Theorem we have:

$$
\begin{aligned}
& \lim _{N \rightarrow \infty} \lim _{m \rightarrow \infty} \frac{1}{m} \sum_{s=0}^{m-1}\left\langle\psi^{(N)}, V_{A}^{s} \hat{f} V_{A}^{-s} \psi^{(N)}\right\rangle \\
& \quad=\lim _{m \rightarrow \infty} \frac{1}{m} \lim _{N \rightarrow \infty} \sum_{s=0}^{m-1}\left\langle\psi^{(N)}, V_{A}^{s} \hat{f} V_{A}^{-s} \psi^{(N)}\right\rangle=\int_{\mathbb{T}^{2}} f d \mu,
\end{aligned}
$$

and moreover

$$
\begin{aligned}
\lim _{l \rightarrow \infty} & \lim _{N \rightarrow \infty} \lim _{m \rightarrow \infty} \frac{1}{m} \sum_{s=0}^{m-1}\left\langle\psi^{(N)}, V_{A}^{l} \hat{f}(s) V_{A}^{-l} \hat{g}(s) \psi^{(N)}\right\rangle \\
\quad & =\lim _{l \rightarrow \infty} \lim _{m \rightarrow \infty} \frac{1}{m} \lim _{N \rightarrow \infty} \sum_{s=0}^{m-1}\left\langle\psi^{(N)}, V_{A}^{l} \hat{f}(s) V_{A}^{-l} \hat{g}(s) \psi^{(N)}\right\rangle \\
& =\int_{\mathbb{T}^{2}} f d \mu \cdot \int_{\mathbb{T}^{2}} g d \mu
\end{aligned}
$$

Remarks.

(1) The Main Theorem is the result analogous to those of [S, CdV, HMR, Z] recalled before, in a stronger form because it holds for all eigenfunctions. The methods of proof are however entirely different from those of [CdV, HMR, Z], because in this case the canonical quantization procedure (Sect. 3) does not preserve positivity and this prevents the direct utilization of the classical ergodicity property. 
(2) Formulae (1.1), (1.2) and the Main Corollary show that, although the quantum system is neither mixing nor ergodic, both these properties are recovered at the classical limit.

\section{Hyperbolic Automorphisms of $\mathbb{T}^{2}$. Classical Properties}

In this section we first recall the basic properties of the hyperbolic automorphisms of $\mathbb{T}^{2}$ implemented by the group of $2 \times 2$ matrices of the form (1.3) and then prove some results concerning the equidistribution of the periodic orbits which might be of some interest in their own, beyond their essential importance in the discussion of the classical limit.

2.1 Koopman Operator on Invariant Lattices and Periodic Orbits. Consider the dynamical system $(M, T, \mu)$ defined in (1), Sect. 1 . The condition $|\operatorname{Tr} A|>2$ makes this dynamical system an Anosov one and hence, in particular, ergodic and mixing with respect to $\mu$. First recall that an orthonormal basis in $L^{2}\left(\mathbb{T}^{2}, d \mu\right)$ is given by the set

$$
D=\left\{T(n)=e^{2 \pi i\langle n, x\rangle} \mid n \in \mathbb{Z}^{2}\right\} .
$$

Consider then in $L^{2}\left(\mathbb{T}^{2}, d \mu\right)$ the unitary Koopman operator $\mathscr{U}_{A}$ defined by

$$
\left(\mathscr{U}_{A} f\right)(x):=f(A x)=\sum_{n \in \mathbb{Z}^{2}} f_{n} T\left(A^{t} n\right)
$$

and recall (see e.g. [AA]) that $T$ is ergodic iff 1 is a simple eigenvalue of $\mathscr{U}_{A}$, i.e., if there is $h \in L^{2}\left(\mathbb{T}^{2}, d \mu\right)$ such that $\mathscr{U}_{A} h=h$, then $h$ is constant (almost everywhere w.r.t. $\mu$ ). Moreover, it is mixing iff, for any pair $f, g \in L^{2}\left(\mathbb{T}^{2}, d \mu\right)$,

$$
\lim _{k \rightarrow \infty}\left\langle\mathscr{U}_{A}^{k} f, g\right\rangle=\langle f, 1\rangle\langle 1, g\rangle .
$$

This property makes $\sigma\left(\mathscr{U}_{A}\right)$ continuous on the unit circle, but for eigenvalue 1 .

There is another important property of $A$ which will be largely used in the sequel. We say that $x \in X$ is a periodic point of $A$, of period $n$, if it is a fixed point of $A^{n}$, i.e. $A^{n} x=x$. We denote by $\mathrm{Fix}_{n}$ the set of such points. It is then easy to see that the set of periodic points of $A$ is dense in $\mathbb{T}^{2}$, because it coincides with the subset of $\pi^{2}$ formed by all points having rational coordinates.

In particular, consider any point on $\mathbb{T}^{2}$ having coordinates $\left(r / N, r^{\prime} / N\right)$, with $r, r^{\prime}, N \in \mathbb{N}$ and $0 \leqq r, r^{\prime}<N$. There are exactly $N^{2}$ points of this type and they belong to the $N \times N$ subgroup of $\mathbb{T}^{2}$ given by:

$$
\mathbb{L}_{N}:=\left\{(q, p) \in \mathbb{T}^{2} \mid N q, N p \in \mathbb{Z}\right\} .
$$

It is immediate to realize that $\mathbb{L}_{N}$ is invariant under the action of $A$, so that any point in $\mathbb{L}_{N}$ is periodic with period $\leqq N^{2}$. The origin is the only fixed point of $A$. Of course, any point $x \in \mathrm{Fix}_{n}$ belongs to a periodic orbit whose period divides $n$. This means that $\mathbb{L}_{N}$ splits into periodic orbits (which in general may have different periods) of $A$.

We now consider once again the atomic measure $\mu_{N}(q)$ on the circle defined by (1.5) and characterize the spectrum of $\mathscr{U}_{A}$ when acting on $L^{2}\left(\mathbb{T}^{2}, d \mu_{N} \times d \mu_{N}\right)$ instead of $L^{2}\left(\pi^{2}, d \mu\right)$. Let $M_{N}$ be the number of distinct periodic orbits of $A^{t}$ which live on $\mathbb{L}_{N} \backslash\{0,0\}$. This number is the same as that corresponding to $A$ (see [I]). Let $\gamma \subset \mathbb{L}_{N}$ 
be any one of such orbits with period $p(\gamma)$ and $x=\left(r_{1} / N, r_{2} / N\right) \in \gamma$. Then, associated to each orbit $\gamma$ there are $p(\gamma)$ linearly independent vectors in $\mathbb{C}^{N^{2}}$ given by:

$$
f_{l}(k)=\sum_{s=0}^{p(\gamma)-1} \lambda_{l}^{-s} \exp \frac{2 \pi i}{N}\left\langle\left(A^{t}\right)^{s} r, k\right\rangle \quad r=\left(r_{1}, r_{2}\right), k \in \mathbb{Z}_{N}^{2},
$$

where $\lambda_{l}=e^{-2 \pi i l / p(\gamma)}$ and $l=0, \ldots, p(\gamma)-1$, and they satisfy

$$
\mathscr{U}_{A} f_{l}(k)=\lambda_{l} f_{l}(k) \text {. }
$$

Thus, there are $N^{2}-1$ eigenvectors of $\mathscr{U}_{A}$ of the form (2.5) which, together with the constant function 1 , provide a canonical basis of $L^{2}\left(\mathbb{T}^{2}, \mu_{N} \times \mu_{N}\right)$. Among them, there are exactly $M_{N}$ non-constant functions which are invariant. This is in account of the fact the dynamical system $\left(\mathbb{T}^{2}, A, \mu_{N} \times \mu_{N}\right)$ is not ergodic: the invariant measure $\mu_{N} \times \mu_{N}$ obviously admits a decomposition into invariant ergodic measures of the type

$$
\mu_{\gamma}:=\frac{1}{p(\gamma)} \sum_{j=0}^{p(\gamma)-1} \delta_{A^{i}(x)}: x \in \gamma .
$$

In the rest of the paper we shall mainly consider the case of $N$ prime. In such case, as shown by [PV], all the periodic orbits have the same period, i.e. $p(\gamma)=p(N)$ for any $\gamma \subset \mathbb{L}_{N} \backslash\{0,0\}$. The relation among $p(N), M_{N}$ and $N$ clearly is:

$$
p(N) \cdot M_{N}=N^{2}-1 \text {. }
$$

We can now sum up the above arguments in the following way:

Proposition 2.1. Let $N$ be a prime number and let $p(N)$ be the period of the cycles living on $\mathbb{L}_{N} \backslash\{0,0\}$. Then $\sigma\left(\mathscr{U}_{A}\right)$ is given by the eigenvalues

$$
\lambda_{l}=e^{2 \pi i l / p(N)} \quad l=0,1, \ldots, p(N)-1 .
$$

To each $\lambda_{l}$ is associated an eigenspace $E_{l}$ of non-constant functions to which all the periodic orbits of $\mathbb{L}_{N} \backslash\{0,0\}$ contribute. Accordingly, the following decomposition holds:

$$
L^{2}\left(\mathbb{T}^{2}, \mu_{N} \times \mu_{N}\right)=\hat{1} \oplus\left(\bigoplus_{l=0}^{p(N)-1} E_{l}\right)
$$

where:

$\hat{1}$ is the one-dimensional subspace spanned by the function 1 and $\operatorname{dim}\left(E_{l}\right)=$ $M_{N} \forall l=0, \ldots, p(N)-1$.

2.2. The Case of N Prime: Structure and Uniform Distribution of Periodic Orbits. We specialize now to the sublattices $\mathbb{L}_{N}$ with $N$ prime defined by (2.6). Since $\mathbb{Z}_{N}$ becomes a finite field, this amounts to operate on a $\bmod N$ arithmetics. Now, following [DE], for $(p, q) \in \mathbb{Q}_{N} \times \mathbb{Q}_{N}=\mathbb{L}_{N}, \mathbb{Q}_{N}=\left\{x: x=\frac{r}{N}, r=0, \ldots, N-1\right\}$ and $l \in \mathbb{Z}_{N}, k \in \mathbb{Z}_{N} \cup\{\infty\}=\widetilde{\mathbb{Z}}_{N}$, let us define the family of subsets $\Lambda_{k, l} \subset \mathbb{L}_{N}$ ("straight lines of slope $2 k ")$ :

$$
\left\{\begin{array}{l}
\Lambda_{k, l}=\left\{(p, q) \mid p-2 k q+\frac{l}{N}(\bmod N), q \in \mathbb{Q}_{N}\right\}, \quad \mathrm{k} \neq \infty \\
\Lambda_{\infty, l}=\left\{(p, q) \mid q=\frac{l}{N}, p \in \mathbb{Q}_{N}\right\} \\
\Lambda_{k, 0}:=\Lambda_{k} .
\end{array}\right.
$$


Remark that $k=\infty$ corresponds to the vertical "line" (" $q=$ const."). Notice also that $\Lambda_{k, l}=\Lambda_{k^{\prime}, l^{\prime}}$ if and only if $k=k^{\prime}, l=l^{\prime}$. Recalling that the prime number $N$ is splitting if there is $n \in \mathbb{Z}_{N}$ such that $(\operatorname{Tr} A)^{2}-4=n^{2} \bmod N$, and is otherwise inert, we have

\section{Lemma 2.1.}

(a) Straight lines are mapped into each other under $A$, i.e. $\forall(k, l)$ there are $\left(k^{\prime}, l^{\prime}\right)$ such that $A \Lambda_{k, l}=\Lambda_{k^{\prime}, l^{\prime}}$. Moreover $A \Lambda_{k}=\Lambda_{k^{\prime}}$.

Let $N$ be splitting, i.e. $(\operatorname{Tr} A)^{2}-4=n^{2} \bmod N$ for some $n \in \mathbb{Z}_{N}$. Then there exists $m \in \mathbb{N}$ such that:

(b) $p(N)=\frac{N-1}{m}$, and there are exactly $m(N+1)$ orbits of period $p(N)$.

(c) The lines $\Lambda_{ \pm \bar{k}}=\{(p, q) \mid p= \pm 2 \bar{k} q\} \subset \mathbb{L}_{N}$ are invariant, i.e. $A \Lambda_{ \pm \bar{k}}=\Lambda_{ \pm \bar{k}}$, where $4 \bar{k}=n \bmod N$.

(d) Both $\Lambda_{\bar{k}}$ and $\Lambda_{-\bar{k}}$ are unions of periodic orbits. The total number of such periodic orbits is $2 \mathrm{~m}$.

If $N$ is inert, then

(e) $p(N)=\frac{N+1}{m}$, and there are $m(N-1)$ orbits of period $p(N)$.

Remark. Let $N$ be splitting. Then the $2 m$ orbits of Assertion (d) coincide with the ideal orbits of [BV, PV]. Consequently, the remaining $m(N-1)$ are the free ones. If $N$ is inert, all orbits are free (see [BV, PV]).

Proof. First remark that the subsets $\Lambda_{k}$ can be described as orbits of the natural $\mathbb{Z}_{N}$ multiplicative action on $\mathbb{L}_{N}$. In fact, for each $x \in \mathbb{L}_{N} \backslash\{0,0\}$ let us define

$$
\Lambda(x)=\left\{u x \mid u \in \mathbb{Z}_{N}\right\} .
$$

Clearly $A \Lambda(x)=\Lambda(A x)$. Moreover $\Lambda(x) \cap \Lambda(y) \neq\{0,0\}$ is equivalent to $y=v x$ for some $v \in \mathbb{Z}_{N}$ which is in turn equivalent to $\Lambda(x)=\Lambda(y)$. This means that we have $N+1=\frac{N^{2}-1}{N-1}$ distinct subsets of the form $\Lambda(x)$. Moreover it is trivial to see that, for any $x \in \mathbb{L}_{N} \backslash\{0,0\}$, there exists a unique $k \in \widetilde{\mathbb{Z}}_{N}$ such that $\Lambda(x)=\Lambda_{k}$. The subsets $\Lambda_{k, l}$ are now obtained by composing the $\mathbb{Z}_{N}$ action on $\mathbb{L}_{N}$ with constant translations. This proves (a).

For the remaining assertions see [PV] ((b)) and (e)) and [BV, DE] ((c) and (d)).

Lemma 2.2. Let $\mathbb{M}_{N}$ be the complement of $\mathbb{L}_{N} \backslash\{0,0\}$ with respect to the $2 m$ possible ideal orbits. Then

(a) If $p(N)$ is odd, there are $k_{0} \neq k_{1} \ldots \neq k_{m-1} \in \mathbb{Z}_{N}$ such that

$$
\bigcup_{j=0}^{m-1} \bigcup_{s=0}^{p-1} A^{s} \Lambda_{k_{j}}=\mathbb{M}_{N}
$$

(b) If $p(N)$ is even, $p=2 q$, there are $k_{0} \neq k_{1} \ldots \neq k_{m-1} \in \mathbb{Z}_{N}, i_{0} \neq i_{1} \ldots \neq i_{m-1} \in$ $\mathbb{Z}_{N}$ such that

$$
\left(\bigcup_{j=0}^{m-1} \bigcup_{s=0}^{q-1} A^{s} \Lambda_{k_{j}}\right) \bigcup\left(\bigcup_{j=0}^{m-1} \bigcup_{s=0}^{q-1} A^{s} \Lambda_{i,}\right)=\mathbb{M}_{N}
$$


Remark. Consider on $\mathbb{M}_{N}$ the straight line $p=2 \mathrm{kq}$. For any fixed $q_{0} \in \mathbb{Q}_{N}$, the map $\varphi_{u}\left(q_{0}, 2 k q_{0}\right)=u\left(q_{0}, 2 k q_{0}\right), u \in \mathbb{Z}_{N}$ yields all points of the line. Since any two points on the line belong to distinct periodic orbits, given a periodic orbit we can generate all others (distinct from the $2 m$ possible ideal ones, assuming without loss $p(N)$ odd) just by considering all iterates of all points having the form $\varphi_{u}\left(q_{0}, 2 k_{j} q_{0}\right): u \in \mathbb{Z}_{N}$, $j=0, \ldots, m-1$, where $\left(q_{0}, 2 k q_{0}=p_{0}\right)$ is the intersection of the given orbit with $\Lambda_{k}$. Namely we have

$$
\mathbb{M}_{N}=\bigcup_{u \in \mathbb{Z}_{N}^{*}} \bigcup_{j=0}^{m-1} \bigcup_{s=0}^{p-1} u A^{s}\left(q_{0}, 2 k_{j} q_{0}\right) .
$$

Proof. Suppose $A^{s} \Lambda(x)=\Lambda(x)$ for some $s, s=1, \ldots, p$. Then either $A^{s} \neq \pm I_{d}$ $\bmod N$ or $A^{s}= \pm I_{d} \bmod N$. In the first case $\Lambda(x)$ is the union of ideal orbits of $A^{s}$ which are also ideal orbits of $A$; that is, $\Lambda(x)=\Lambda_{ \pm \bar{k}}, \bar{k}$ as in Lemma 2.1(c). In the other case, $s=p$ if $p$ is odd, or $s=q=\frac{p}{2}$ if $p$ is even, and $A^{q}=-I_{d} \bmod N$. Therefore, if $p$ is odd, the disjoint union

$$
\mathscr{S}_{k}=\bigcup_{s=0}^{p-1} A^{s} \Lambda_{k}
$$

is invariant and contains $1+p(N-1)$ points. If $N$ is inert, $p=\frac{N+1}{m}$, and $\mathscr{S}_{k}$ contains exactly $1+\frac{\left(N^{2}-1\right)}{m}$ points independently of $k \in \mathbb{Z}_{N}$. On the other hand, each set $\mathscr{S}_{k}$ is the union of $N-1$ disjoint periodic orbits generated by the orbits $\bigcup_{s=0}^{p-1} A^{s} x$, of any one of the $N-1$ points $x \in \Lambda_{k}, x \neq(0,0)$. Since the total number of orbits is $m(N-1)$, we can immediately conclude that there is at least one choice of indices $k_{0} \neq k_{1} \neq \cdots \neq k_{m-1}$ such that:

$$
\mathbb{M}_{N}=\bigcup_{j=0}^{p-1} \mathscr{S}_{k_{j}}=\bigcup_{j=0}^{m-1} \bigcup_{s=0}^{p-1} A^{s} \Lambda_{k_{\jmath}} .
$$

The extension to $N$ splitting is immediate, and this proves (a).

(b) If $p=2 q$, remark once more that $A^{q} \Lambda_{k}=\Lambda_{k}$ because $A^{q} x=-x \bmod N$ $\forall x \in \mathbb{L}_{N}$, i.e. $\left.A^{q}\right|_{\mathbb{L}_{N}}=-I$. Then the assertion can proved by repeating the above argument.

Let us now turn to the study of the equidistribution properties of periodic orbits living on prime lattices. Under slightly more restrictive assumptions on the sequence of primes and using the number theoretic techniques collected in Appendix A, we are able to prove the equidistribution of all periodic orbit sequences (living on prime lattices), with explicit estimates of the speed of convergence. This result looks somewhat stronger than the equidistribution property on the average (with respect to the measure of maximal entropy) valid for all Anosov systems (see e.g. [PP]). More precisely,

Theorem 2.2. Let $N \in \Gamma, \Gamma$ being any increasing sequence of primes such that $\frac{N}{p(N)}<C$ for some $C$ independent of $N$. Set:

$$
P_{N}:=\left\{\gamma \subset \mathbb{L}_{N} \backslash\{0,0\} \mid \gamma \text { periodic orbit of } A\right\} ; \quad M_{N}:=\# P_{N} .
$$


Then, given any $f \in A\left(\mathbb{T}^{2}\right)$, any sequence $\left\{\gamma_{j(N)}\right\}_{N \in \Gamma}$ such that $\gamma_{j(N)} \in P_{N}, j(N) \in$ $\left\{1, \ldots, M_{N}\right\}$ we have

$$
\lim _{N \rightarrow \infty}\left|\int_{\gamma,(N)} f-\int_{\mathbb{T}^{2}} f d \mu\right|=0 .
$$

Moreover, if $f \in \mathbb{C}^{\infty}\left(\mathbb{T}^{2}\right)$ there are $C>0$ and $\bar{N}>0$ such that, for $N>\bar{N}$ :

$$
\left|\int_{i,(N)} f-\int_{\mathbb{T}^{2}} f d \mu\right|<\frac{C}{\sqrt{N}}\|f\|_{A\left(T^{2}\right)} .
$$

Remark. The condition $N \in \Gamma$ is equivalent to require $m$ bounded with respect to $N$. Since $m p=N \pm 1, m \sim \frac{N}{p}$ which is in turn a constant multiple of the quantum degeneracy, this condition (see the remark after Proposition 3.8 below) means restriction to those values of $N$ for which the quantum degeneracy does not grow at the classical limit $N \rightarrow \infty$

Proof. We shall give the argument for the case of $N$ splitting. This is equivalent to the splitting of the characteristic polynomial over $\mathbb{Z}_{N}$. In particular (see Appendix), if we let $D=\sqrt{\frac{(\operatorname{Tr} A)^{2}}{4}-1} \in \mathbb{Z}_{N}$, then $v=(1, D), w=(1,-D) \in \mathbb{Z}_{N}^{2}$ are the eigenvectors of $A$ acting on $\mathbb{Z}_{N}^{2}$, corresponding to the eigenvalues $\lambda_{N}=\operatorname{Tr} A / 2+D$ and $\lambda_{N}^{-1}=\operatorname{Tr} A / 2-D$, respectively. Now the key point is that the integral of an arbitrary character $e^{i 2 \pi\langle n, x\rangle}$ over a periodic orbit $\gamma \subset \mathbb{L}_{N}$ can be written as a Kloosterman sum restricted to a cyclic subgroup of $\mathbb{Z}_{N}^{*}$ of order $(N-1) / m$. Indeed, for any $\gamma \subset \mathbb{L}_{N}$ and $x \in \gamma$ set $N x=\alpha v+\beta w$, where $\alpha(x), \beta(x) \in \mathbb{Z}_{N}$. Then,

$$
\begin{aligned}
\int_{\gamma \in \mathbb{L}_{N}} e^{i 2 \pi\langle n . x\rangle}=\frac{1}{p} \sum_{s=0}^{p-1} e^{i 2 \pi\left\langle n, A^{s} x\right\rangle} & =\frac{1}{p} \sum_{s=0}^{p-1} e^{\left.\left.\frac{12 \pi}{N}\left(\langle n, x v\rangle_{N}^{s}+\beta w\right\rangle_{N}^{s}\right)\right\rangle} \\
& =\frac{1}{p} \sum_{s=0}^{p-1} e^{\left.\frac{22 \pi}{N}(\gamma\langle n, v\rangle\rangle_{N}^{s}+\beta\langle n, w\rangle \bar{N}^{s}\right)},
\end{aligned}
$$

that is

$$
\begin{aligned}
\int_{\because \subset \mathbb{L}_{N}} e^{i 2 \pi\langle n, x\rangle} & =\frac{1}{m p} \sum_{\xi \in \mathbb{Z}_{N}^{*}} e^{\frac{12 \pi}{N}\left(a \check{c}^{m}+b \xi-m\right)} \\
& =\frac{1}{m p} \sum_{j=0}^{m-1} \sum_{\xi \in \mathbb{Z}_{N}^{*}} \chi_{j}(\xi) e^{\frac{12 \pi}{N}(a \xi+b \xi-1)},
\end{aligned}
$$

where $a=\alpha\langle n, v\rangle, b=\beta\langle n, w\rangle \in \mathbb{Z}_{N}$ and the relation $p=\frac{N-1}{m}$ has been used. The functions $\chi_{j}: j=0, \ldots, m-1$ are the $m$ distinct multiplicative characters of order $m$ over $\mathbb{Z}_{N}$ (see Appendix A, Proposition A.2). Now by the estimate (A.3) we have, for any $n \neq(0,0)(\bmod N)$ :

$$
\left|\int_{\gamma \in \mathbb{L}_{N}} e^{i 2 \pi\langle n, x\rangle}\right| \leqq \frac{\bar{C}}{\sqrt{N}},
$$


where $\bar{C}>0$ is independent of $n$ and of the particular orbit $\gamma \subset \mathbb{L}_{N}$ and is uniformly bounded in $N$ because of the boundedness of $m$. On the other hand, a trivial computation shows that, if $n=(0,0)(\bmod N)$ then

$$
\int_{\gamma \in \mathbb{L}_{N}} e^{i 2 \pi\langle n, x\rangle}=1
$$

Therefore if $f \in A\left(\mathbb{T}^{2}\right), f=\sum_{n \in \mathbb{Z}^{2}} f_{n} e^{i 2 \pi\langle n, x\rangle}$, we can write

$$
\int_{\gamma, j} f-\int_{\mathbb{T}^{2}} f d \mu=\sum_{n \neq(0,0)(\bmod N)} f_{n} \int_{\gamma,(N)} e^{i\langle n, x\rangle}+\sum_{k \neq(0,0)} f_{k N} .
$$

Now, if $f \in A\left(\mathbb{T}^{2}\right)$ the second term of the r.h.s. vanishes as $N \rightarrow \infty$ and the first term also vanishes by the uniform estimate (2.15) on the Kloosterman sums. Moreover if $f \in \mathbb{C}^{\infty}\left(\mathbb{T}^{2}\right)$, the second term vanishes at least as $N^{-1}$ as $N \rightarrow \infty$, and therefore we can conclude that there exists $C>0$ such that if $N>\bar{N}$ :

$$
\left|\int_{\gamma /(N)} f-\int_{\mathbb{T}^{2}} f d \mu\right| \leqq \frac{C}{\sqrt{N}}\|f\|_{A\left(\mathbb{T}^{2}\right)} .
$$

Finally, the case $N$ inert can be treated in a similar way by using the techniques of [PV] and the generalized Kloosterman sums over arbitrary finite fields [Ka1].

\section{Quantization, Discrete Wigner Function and Eigenvector Construction}

3.1 Quantization of the Observables and of the Automorphism. The canonical quantization procedure when the phase space is the torus $\mathbb{T}^{2}$ has been developed in [DE] in complete analogy to the standard quantization in $\mathbb{R}^{2 n}$ via representations of the Heisenberg group $\mathbb{H}_{n}(\mathbb{R})$ (see e.g. [Fo]). According to this procedure the natural objects to look at are:

\section{Definition 3.1.}

(1) $\forall h \in \mathbb{R} \backslash 0, \mathscr{H}_{1}:=\mathbb{H}_{1}(\mathbb{Z})$ is the group topologically equivalent to $\mathbb{Z}^{2} \times \mathbb{R}$ with group law

$$
(n, t)(m, s)=\left(n+m, t+s+\frac{1}{2} \omega(n, m)\right) .
$$

(2) $\mathscr{A}_{h}$ is the unitary $*$-algebra over $\mathbb{C}$ generated by the group

$$
\mathscr{T}_{h}=\{\hat{T}(n)\}_{n \in \mathbb{Z}^{2}},
$$

where

$$
\left\{\begin{array}{l}
\hat{T}(n)^{*}=\hat{T}(-n) \\
\hat{T}(n) \hat{T}(m)=e^{i \pi h \omega(n, m)} \hat{T}(n+m) .
\end{array}\right.
$$

$\mathbb{H}_{1}(\mathbb{Z})$ is the discrete Heisenberg group and $\mathscr{A}_{h}$ the associated discrete Heisenberg algebra. The canonical quantization is obtained upon classification of all finite-dimensional irreducible representations of $\mathscr{A}_{h}$, defined abstractly by the properties (3.1), into the unitary operators acting in the Hilbert spaces $L^{2}\left(S^{1} ; \lambda\right)$, for some measure $\lambda$ on $S^{1}$ to be determined. It is proved in [DE] that finite-dimensional representations exist if and only if $h=p / N$ (for the sake of simplicity we will 
henceforth assume $p=1$ ). To recall the basic facts about these representations, first consider the generators of the algebra, defined as:

$$
t_{1}:=\hat{T}(1,0) ; \quad t_{2}:=\hat{T}(0,1) .
$$

In fact, if $n=\left(n_{1}, n_{2}\right)$ we have:

$$
\left\{\begin{array}{l}
\hat{T}(n)=\exp \left[\pi i \frac{n_{1} n_{2}}{N}\right] t_{2}^{n_{2}} t_{1}^{n_{1}} \\
t_{2} t_{1}=e^{-2 i \pi h} t_{1} t_{2} .
\end{array}\right.
$$

If $h=1 / N$ then $t_{1}^{N}$ and $t_{2}^{N}$ are the generators of the center and each one is mapped into a unitary scalar multiple of the identity by any irreducible representation. The two corresponding phases define the representations which are always $N$-dimensional. In other words, the unique infinite dimensional unitary representation of the standard Heisenberg group splits into a direct integral (over the two torus) of finite dimensional, non-equivalent, unitary representations.

Consider now, once again, the $N$ dimensional Hilbert space $L^{2}\left(S^{1}, \mu_{N}\right)$, where $\forall h=N^{-1}, \mu_{N}(x)$ is the atomic measure on the circle defined by (1.5). Let us remark that the vectors $|k\rangle=\Psi_{k}(x)=\delta_{k / N}^{x}$ for $k=0,1, \ldots, N-1$ are a basis of the Hilbert space $L^{2}\left(S^{1}, \mu_{N}\right)$, and recall that inner product between $\psi, \phi \in L^{2}\left(S^{1}, \mu_{N}\right)$ is given by:

$$
\langle\psi, \phi\rangle:=\frac{1}{N} \sum_{l=0}^{N-1} \bar{\psi}\left(\frac{l}{N}\right) \phi\left(\frac{l}{N}\right)=\int_{S^{1}} \bar{\psi}(x) \phi(x) d \mu_{N}(x) .
$$

On the other hand, the action of the Fourier transformation on $L^{2}\left(S^{1}, \mu_{N}\right)$ is:

$$
\left(\mathscr{F}_{N} \psi\right)_{m}:=\frac{1}{\sqrt{N}} \sum_{n=0}^{N-1} \exp \left(\frac{2 i \pi m n}{N}\right) \psi_{n} .
$$

Writing

$$
\mathbb{Q}_{N}=\{0,1 / N, 2 / N, \ldots,(N-1) / N\} ; \quad \mathbb{Z}_{N}:=\mathbb{Z} / N \mathbb{Z}=\{0,1,2, \ldots, N-1\}
$$

we can identify $L^{2}\left(S^{1}, \mu_{N}\right)$ with $L^{2}\left(\mathbb{Z}_{N}, \mu_{N}\right)$, where $\psi \in L^{2}\left(\mathbb{Z}_{N}, \mu_{N}\right)$ is a vector in $\mathbb{C}^{N}: \psi=\left(\psi_{0}, \ldots, \psi_{N-1}\right)$.

Now, for any fixed $\theta \in \mathbb{T}^{2}$, the representations of our algebra on $L^{2}\left(S^{1}, \mu_{N}\right)$ are defined by specifying their action on the generators (identified with the corresponding matrices):

$$
\begin{array}{ll}
t_{1}|l\rangle=\exp \left(\frac{2 i \pi\left(\theta_{1}+l\right)}{N}\right)|l\rangle, & t_{1}^{N}=e^{2 \pi i \theta_{1}} \cdot \mathrm{Id}, \\
t_{2}|l\rangle=\exp \left(\frac{2 i \pi \theta_{2}}{N}\right)|l+1\rangle, \quad t_{2}^{N}=e^{2 \pi i \theta_{2}} \cdot \mathrm{Id} .
\end{array}
$$

It is proved in $[\mathrm{DE}]$ that these representations are irreducible, non-equivalent for different values of $\theta$ and moreover that they are the only possible ones.

For any fixed $h=1 / N$ and $\theta \in \mathbb{T}^{2}$ we denote by $\hat{T}_{h, \theta}$ the representation of $\hat{T}(n)$ just recalled:

$$
\widehat{T}_{h, \theta}(n)=e^{\left[\frac{\pi n n_{1} n_{2}}{N}\right]} t_{2}^{n_{2}} t_{1}^{n_{1}}
$$


We quantize any observable $f \in A\left(\mathbb{T}^{2}\right)$,

$$
f=\sum_{n \in \mathbb{Z}^{2}} f_{n} T(n), \quad T(n)=e^{2 \pi i\langle n, x\rangle}
$$

by the correspondence

$$
f \mapsto \hat{f}=\sum_{n \in \mathbb{Z}^{2}} f_{n} \hat{T}(n)
$$

i.e., we associate to $f$ the element of the algebra, denoted by $\hat{f}$, obtained replacing $T(n)$ by $\hat{T}(n)$ into the Fourier expansion.

Let $A \in \mathrm{SL}(2, \mathbb{Z})=\mathrm{Sp}(1, \mathbb{Z})$. To implement the quantum dynamics, consider the new representation defined by $\rho_{A}(n)=\hat{T}_{h, \theta} \circ A^{t}(n)=\hat{T}_{h, \theta}\left(A^{t} n\right): \forall n \in \mathbb{Z}^{2}$. Let us see that $\rho_{A}$ is again an irreducible representation, and thus unitarily equivalent to one of the previous. The unitary intertwining operator realizes the "commutativity between classical evolution and quantization" and thus represents the quantum propagator. More precisely, we have the following

Theorem 3.1. Let $A=\left(\begin{array}{ll}a & b \\ c & d\end{array}\right) \in S L(2, \mathbb{Z})$. Then:

(1) $\rho_{A}(n)=\hat{T}_{h, \theta}\left(A^{t} n\right)$ is again an irreducible representation of $\mathscr{A}_{h}$;

(2) $\forall \theta \in \mathbb{T}^{2}$, there exists a unitary operator $V_{A}(\theta)$ such that, $\forall n \in \mathbb{Z}^{2}$ :

$$
\hat{T}_{h, 0}\left(A^{t} n\right)=V_{A}(\theta)^{-1} \circ \hat{T}_{h, \varphi_{A}(\theta)}(n) \circ V_{A}(\theta),
$$

where

$$
\varphi_{A}(\theta)=A(\theta)+\frac{1}{2}\left(\begin{array}{l}
a b N \\
c d N
\end{array}\right) \bmod 1 .
$$

Proof. To see assertion (1), it is enough to remark that $A$ is a symplectic matrix, i.e. $\omega\left(A^{t} m, A^{t} n\right)=\omega(m, n)$, which implies

$$
\rho_{A}(m) \rho_{A}(n)=\exp \frac{i \pi}{N} \omega(m, n) \rho_{A}(m+n) .
$$

To prove (2), note that the central character of the new representation is completely defined by

(1)

$$
\hat{T}_{h, \theta}\left(A^{t}\left(\begin{array}{l}
N \\
0
\end{array}\right)\right)=e^{\pi i a b N} e^{2 \pi i\left(\theta_{1} a+\theta_{2} b\right)}=\hat{T}_{h, \varphi_{A}(\theta)}\left(\left(\begin{array}{l}
N \\
0
\end{array}\right)\right),
$$

(2)

$$
\widehat{T}_{h, \theta}\left(A^{t}\left(\begin{array}{l}
0 \\
N
\end{array}\right)\right)=e^{\pi i c d N} e^{2 \pi i\left(\theta_{1} c+\theta_{2} d\right)}=\hat{T}_{h, \varphi_{A}(\theta)}\left(\left(\begin{array}{l}
0 \\
N
\end{array}\right)\right),
$$

and therefore the result follows immediately.

Remarks.

(1) In the case of $\mathbb{R}^{2 n}$ we have only one irreducible representation of the Heisenberg group for any given value of the central character (Stone-Von Neumann Theorem) and for any given automorphism $A \in \mathrm{Sp}(n, \mathbb{R})$ we obtain a unitary 
operator $V_{A}$ (defined up to a phase) such that $V_{A B}= \pm V_{A} V_{B}$. The (double valued) map $A \longrightarrow \pm V_{A}$ is the so-called metaplectic representation for $\operatorname{Sp}(n, \mathbb{R})$ (which is a well defined representatin in the double cover of $\operatorname{Sp}(n, \mathbb{R})[\mathrm{F}]$ ).

(2) The unitary operator $V_{A}(\theta)$ in (3.10) is by definition the quantization of the map $A$, i.e. the unit-time propagator describing the quantum discrete evolution.

(3) If $\varphi_{A}(\theta)=\theta$ for some $\theta$, then $\hat{T}_{h, \theta^{\circ}} \circ A^{t}=V_{A}(\theta)^{-1} \circ \hat{T}_{h, \theta^{\circ}} \circ V_{A}(\theta)$. In particular if $A=\left(\begin{array}{ll}\text { even } & \text { odd } \\ \text { odd } & \text { even }\end{array}\right)$ or $A=\left(\begin{array}{ll}\text { odd } & \text { even } \\ \text { even } & \text { odd }\end{array}\right)$, we can choose $\theta=(0,0)$. These maps are exactly those called "quantizable" by Berry and Hannay [BH]. An example of a map with a non-trivial $\theta$ is given by the usual cat map $A=\left(\begin{array}{ll}2 & 1 \\ 1 & 1\end{array}\right)$ for which we have $\varphi_{A}(\theta)=\theta$ if $\theta=\left(\frac{1}{2}, \frac{1}{2}\right)$.

(4) If, in particular, $A=J=\left(\begin{array}{rr}0 & 1 \\ -1 & 0\end{array}\right)$, then it is easily seen that

$$
V_{A}(\theta)=\mathscr{F}_{N} .
$$

While the solution of (3.10) can be obtained $\forall A \in \operatorname{SL}(2, \mathbb{Z})$ to yield the explicit formula for the propagator $V_{A}(\theta)$ (see [DE]), for the sake of simplicity we limit ourselves here to the matrices of the form

$$
A=\left(\begin{array}{cc}
2 g & 1 \\
4 g^{2}-1 & 2 g
\end{array}\right)
$$

with $g \in \mathbb{N}$. These matrices are a subset of the Berry-Hannay ones. In this particular case the explicit form of the propagator is:

$$
\left\{\begin{array}{l}
V_{A}\left|q_{2}\right\rangle=\sum_{q_{1}=0}^{N-1} V_{A}\left(q_{2}, q_{1}\right)\left|q_{1}\right\rangle \\
V_{A}\left(q_{1}, q_{2}\right)=\frac{C_{N}}{\sqrt{N}} \exp \frac{2 \pi i}{N}\left(g q_{1}^{2}-q_{1} q_{2}+g q_{2}^{2}\right),
\end{array}\right.
$$

where $C_{N}$ is an arbitrary phase factor. Now consider $A \in \operatorname{SL}(2, \mathbb{Z})$ fixed as above. For any fixed $h=1 / N$, let us abbreviate $V_{A}(\theta)$ by $V_{A}$ and $\hat{T}_{h, \theta}(n)$ by $\hat{T}(n)$. Therefore if $A^{p}=I_{d} \bmod N$, that is if $A^{p}$ is the identity when restricted to the points on the torus with rational coordinates of denominator $N$, then:

$$
V_{A}^{p}=e^{2 \pi i \sigma} I_{d}
$$

for some constant phase $\sigma$ (depending on $N$ ).

This restricts the $N$ eigenvalues of $V_{A}$ to lie on the $p$ possible sites:

$$
\left\{\exp \left[\frac{2 \pi i(m+\sigma)}{p}\right] \mid 0 \leqq m \leqq p-1\right\} .
$$

In general $p(N) \neq N$, that is, there is no one-to-one correspondence between eigenvalues and sites. Typically, for a hyperbolic map, there are both unoccupied and multiply occupied sites and this distribution follows the highly irregular behavior of $p=p(N)$ as a function of $N[\mathrm{~K} 2, \mathrm{~K} 3]$. 
Note that if $\psi \in L^{2}\left(\mathbb{Z}_{N}, \mu_{N}\right)$ with $\psi=\sum_{l=0}^{N-1} a_{l}|l\rangle$, then, supposing $N$ prime and $\theta=(0,0)$ fixed, $\forall n=\left(n_{1}, n_{2}\right) \in \mathbb{Z}^{2}$ we have the expression

$$
(\widehat{T}(n) \psi)(k)=e^{-\frac{i \pi n n n_{2}}{N}} e^{\frac{2 i \pi n n_{1} k}{N}} \psi\left(k-n_{2}\right),
$$

where of course, $k-n_{2}=k-n_{2} \bmod N$ and where we interpret, as already mentioned, $\psi$ as a function on $\mathbb{Q}_{N} \simeq \mathbb{Z}_{N}$, i.e. $\psi\left(\frac{s}{N}\right):=\psi(s)=a_{s}$.

3.2 The Discrete Wigner Function and its Properties. Let us now proceed to the construction of the discrete Wigner distribution. In complete analogy with [Fo], Sect. 1.8, let us first define the discrete Fourier-Wigner transform $V(\phi, \psi)(n)$ of any pair of vectors $(\phi, \psi) \in L^{2}\left(\mathbb{Z}_{N}, \mu_{N}\right)$ :

$$
V(\phi, \psi)(n)=\langle\phi, \hat{T}(n) \psi\rangle=e^{-\frac{\imath \pi n n_{2}}{N}} \cdot \frac{1}{N} \sum_{k \in \mathbb{Z}_{N}} e^{\frac{2 u n n_{1} k}{N}} \psi\left(k-n_{2}\right) \bar{\phi}(k) .
$$

Since $N \in \mathbb{N}$ is prime we can also write

$$
V(\phi, \psi)(n)=\frac{1}{N} \sum_{k \in \mathbb{Z}_{N}} e^{\frac{2 i \pi n_{1} k}{N}} \psi\left(k-\frac{n_{2}}{2}\right) \bar{\phi}\left(k+\frac{n_{2}}{2}\right),
$$

where $\frac{x}{2}$ is the unique integer in the field $\mathbb{Z}_{N}$ such that $2 \cdot \frac{x}{2}=x \bmod N$.

Remark also that the periodicity of $\widehat{T}(n)$, namely $\widehat{T}(n)=\hat{T}(n+N \cdot k)$, implies $\forall k, n \in \mathbb{Z}^{2}$ the same property on $V(\phi, \psi)(n)$ :

$$
V(\phi, \psi)(n+N \cdot k)=V(\phi, \psi)(n) .
$$

The main relevant properties of the Fourier-Wigner transform are:

Proposition 3.1. Let $n, l, m \in \mathbb{Z}_{N}$. Then

(1) $V(\hat{T}(n) \phi, \hat{T}(m) \psi)(l)=e^{-\frac{l \pi}{N} \omega(n, l)} \cdot e^{\frac{l \pi}{N} \omega(l-n, m)} \cdot V(\phi, \psi)(l+m-n)$.

In particular:

(2) $V(\hat{T}(n) \phi, \psi)(l)=e^{-\frac{\imath \pi}{N} \omega(n, l)} \cdot V(\phi, \psi)(l-n)$,

(3) $V(\phi, \hat{T}(m) \psi)(l)=e^{\frac{i \pi}{N} \omega(l, m)} \cdot V(\phi, \psi)(l+m)$,

(4) $V(\hat{T}(n) \phi, \hat{T}(n) \psi)(l)=e^{-\frac{2 i \pi}{N} \omega(n, l)} \cdot V(\phi, \psi)(l)$.

Proof.

$$
\begin{aligned}
V(\widehat{T}(n) \phi, \hat{T}(m) \psi)(l) & =\langle\hat{T}(n) \phi, \hat{T}(l) \hat{T}(m) \psi\rangle \\
& =\langle\phi, \hat{T}(-n) \hat{T}(l) \hat{T}(m) \psi\rangle \\
& =e^{-\frac{l \pi}{N} \omega(n, l)}\langle\phi, \hat{T}(l-n) \hat{T}(m) \psi\rangle \\
& =e^{-\frac{2 \pi}{N} \omega(n, l)} \cdot e^{\frac{l \pi}{N} \omega(l-n, m)}\langle\phi, \hat{T}(l+m-n) \psi\rangle .
\end{aligned}
$$

Given $A \in \operatorname{SL}(2, \mathbb{Z})$, with $\varphi_{A}(\theta)=\theta$, denote once more by $e_{0}, \ldots, e_{N-1}$ an orthonormal set of eigenfunctions of $V_{A}$, i.e.

$$
V_{A} e_{k}=e^{\frac{21 \pi}{N} \lambda_{k}} e_{k}
$$


and $\forall k, l \in \mathbb{Z}_{N}$, let us introduce the following family of $N^{2}$ functions:

$$
V_{l, k}(n):=V\left(e_{l}, e_{k}\right)(n) .
$$

Set moreover $V_{l}:=V_{l, l}$. Then we have:

\section{Lemma 3.1.}

$$
\sum_{l=0}^{N-1} V_{l}(n)=\operatorname{Tr}(\hat{T}(n))=N \cdot \delta_{n}^{(0,0)} .
$$

Proof. Note that $\operatorname{Tr}\left(t_{1}\right)=\operatorname{Tr}\left(t_{2}\right)=0$. Moreover, since

$$
t_{2}^{n_{2}} t_{1}^{n_{1}}=e^{-\frac{2 i \pi}{N} n_{2} n_{1}} t_{1}^{n_{1}} t_{2}^{n_{2}}
$$

the assertion follows by the commutativity of the trace.

We are now in position to define the discrete Wigner transform which also in this case is a map from pairs of functions in the Hilbert space into the phase-space functions, namely:

Definition 3.2. Let $\phi, \psi \in L^{2}\left(\mathbb{Z}_{N}, \mu_{N}\right),(q, p) \in \mathbb{T}^{2}$. Then their discrete Wigner transform is the function defined as

$$
W(\phi, \psi)(q, p)=\sum_{n_{1}, n_{2} \in \mathbb{Z}_{N}}\langle\phi, \hat{T}(n) \psi\rangle e^{-2 i \pi\left(n_{1} q+n_{2} p\right)} .
$$

Since the Wigner functions have to be integrated against the measures $\mu_{N}$ on $S^{1}$ or $\mu_{N} \times \mu_{N}$ on $\mathbb{T}^{2}$, we are interested only in the values they assume on the lattice $\mathbb{L}_{N}$, and therefore we use the notation:

$$
W(\phi, \psi)(s, r):=W(\phi, \psi)\left(\frac{s}{N}, \frac{r}{N}\right),
$$

$\forall s, r \in \mathbb{Z}_{N}$. Now, using the inverse of the discrete Fourier transform we get $\left(x=(q, p)=(r / N, s / N) \in \mathbb{L}_{N}\right)$

$$
\begin{aligned}
W(\phi, \psi)(s, r) & =\sum_{k \in \mathbb{Z}_{N}} e^{-\frac{2 i \pi k r}{N}} \phi\left(s-\frac{k}{2}\right) \bar{\psi}\left(s+\frac{k}{2}\right) \\
& =N \int e^{-2 i \pi N 2 p q^{\prime}} \phi\left(q-q^{\prime}\right) \bar{\psi}\left(q+q^{\prime}\right) d \mu_{N}\left(q^{\prime}\right) .
\end{aligned}
$$

In particular for $\psi=\phi$ we obtain the formula for the Wigner distribution

$$
W_{\phi}\left(m_{1}, m_{2}\right):=W(\phi, \phi)\left(m_{1}, m_{2}\right)=\sum_{k \in \mathbb{Z}_{N}} e^{-\frac{2 \pi n 2 k m_{2}}{N}} \phi\left(m_{1}-k\right) \bar{\phi}\left(m_{1}+k\right) .
$$

As a direct consequence of the Cauchy-Schwartz inequality applied to the previous equation, we have

Lemma 3.2. For any pair of normalized vectors $\phi, \psi \in L^{2}\left(\mathbb{Z}_{N}, \mu_{N}\right)$,

$$
\sup _{x \in \mathbb{L}_{N}}|W(\phi, \psi)(x)| \leqq N .
$$

We can summarize the basic properties of $W(\phi, \psi)$ in the following proposition, whose verification is omitted because it is an easy exercise based on [F, Sect. 1.8]. 


\section{Proposition 3.2.}

(1)

$$
W(\hat{T}(n) \phi, \hat{T}(m) \psi)(l)=e^{i \sigma(n, l, m)} \cdot W(\phi, \psi)\left(l_{1}-\frac{n_{2}-m_{2}}{2}, l_{2}+\frac{n_{1}+m_{1}}{2}\right),
$$

where

$$
e^{i \sigma(n, l, m)}=e^{\frac{2 i \pi(\omega)(n, m)}{N}} \cdot e^{-\frac{2 i \pi}{N} \omega(n-m, l)} .
$$

(2) $W\left(\mathscr{F}_{N} \phi, \mathscr{F}_{N} \psi\right)\left(l_{1}, l_{2}\right)=W(\phi, \psi)\left(l_{2},-l_{1}\right)$,

(3) $W(\psi, \phi)=\bar{W}(\phi, \psi)$,

(4) $\int W_{\phi}(q, p) d \mu_{N}(q)=\frac{1}{N} \sum_{s \in \mathbb{Z}_{N}} W_{\phi}(s / N, p)=\left|\mathscr{F}_{N} \phi(p)\right|^{2}$,

(5) $\int W_{\phi}(q, p) d \mu_{N}(p)=\frac{1}{N} \sum_{r \in \mathbb{Z}_{N}}^{s \in \mathbb{Z}_{N}} W_{\phi}(q, r / N)=|\phi(q)|^{2}$,

(6) $W_{i \phi}=W_{\phi} \quad \forall \lambda \in S^{1}$,

(7)

$$
\left.\left\langle W\left(\psi_{1}, \phi_{1}\right), W\left(\psi_{2}, \phi_{2}\right)\right\rangle\right|_{L^{2}\left(\mathbb{T}^{2}, \mu_{N} \times \mu_{N}\right)}=\left.\left.N^{2}\left\langle\psi_{1}, \psi_{2}\right\rangle\right|_{L^{2}\left(S^{1}, \mu_{N}\right)}\left\langle\phi_{1}, \phi_{2}\right\rangle\right|_{L^{2}\left(S^{1}, \mu_{N}\right)}
$$

(Moyal's identity).

In particular from the first equation we have

$$
W(\hat{T}(n) \phi, \hat{T}(n) \psi)(l)=W(\phi, \psi)\left(l_{1}-n_{2}, l_{2}+n_{1}\right) .
$$

Now it is easy to relate the "quantum" evolution given by the unitary operator $V_{A}$ to the symplectic action of $A$ on the Wigner functions.

Theorem 3.2. Under the previous notation, we have

(1) $V\left(V_{A} \phi, V_{A} \psi\right)=V(\phi, \psi) \circ A^{t}$,

(2) $W\left(V_{A} \phi, V_{A} \psi\right)=W(\phi, \psi) \circ A^{-1}$.

Proof. Assume to simplify the exposition $\varphi_{A}((0,0))=(0,0)$. Then:

$$
\begin{aligned}
V\left(V_{A} \phi, V_{A} \psi\right) & =\left\langle V_{A} \phi, \hat{T}(n) V_{A} \psi\right\rangle=\left\langle\phi, V_{A}^{-1} \hat{T}(n) V_{A} \psi\right\rangle \\
& =\left\langle\phi, \hat{T}\left(A^{t} n\right) \psi\right\rangle=V(\phi, \psi) \circ A^{t} .
\end{aligned}
$$

On the other hand

$$
\begin{aligned}
W\left(V_{A} \phi, V_{A} \psi\right)(q, p) & =\sum_{n \in \mathbb{Z}_{N}^{2}} V\left(V_{A} \phi, V_{A} \psi\right) e^{2 i \pi\left(q n_{1}+p n_{2}\right)} \\
& =\sum_{n \in \mathbb{Z}_{N}^{2}} V(\phi, \psi)\left(A^{t} n\right) e^{2 i \pi\left(q n_{1}+p n_{2}\right)} \\
& =W(\phi, \psi)\left(A^{-1}(q, p)\right),
\end{aligned}
$$

and this proves the assertion.

The Wigner function allows us to define a family of quasi-invariant functions on the lattice, that is a set of eigenvectors for the Koopman operator acting on $L^{2}\left(\mathbb{T}^{2}, \mu_{N} \times \mu_{N}\right)$, namely: 
Proposition 3.3. Let $\left\{W_{k, l}\right\}$ be the family of $N^{2}$ functions on the torus given by

$$
W_{k, l}(x)=W\left(e_{k}, e_{l}\right)(x)
$$

(with $\left.W_{l}:=W_{l . l}\right)$. Then

(1) $\forall x \in \mathbb{L}_{N}$ :

$$
W_{k, l}(A x)=e^{2 i \pi\left(\lambda_{1}-\lambda_{l}\right)} W_{k, l}(x) .
$$

(2) If $\phi$ is an eigenvector for $V_{A}$ then $W_{\phi}$ is an eigenvector with eigenvalue 1 of (the adjoint of ) the Koopman operator defined by $A$, i.e.

$$
W_{V_{A} \phi}=W_{\phi}=\mathscr{U}_{A}^{*} W_{\phi} .
$$

Proof. Formula (3.22) is an immediate consequence of Definition (3.16) and of Theorem 3.2. Moreover, if $\lambda_{l}=\lambda_{k}$, i.e. if $l=k$ or if $e_{l}$ and $e_{k}$ belong to the same eigenspace, we obtain the eigenspace of the Koopman operator of eigenvalue one. Using this and Assertion (6) of Proposition 3.2 we immediately get (3.23) as well.

Remarks.

(1) According to the discussion given at the end of Sect. 2, we recover the relation between the degeneracy of the eigenvalues of the quantum propagator $V_{A}$ and the dimension of the invariant eigenspace of the Koopman operator acting on $L^{2}\left(\mathbb{T}^{2}, \mu_{N} \times \mu_{N}\right)[\mathrm{BE}, \mathrm{E} 1]$ :

$$
\sum_{i=1}^{p(N)} d_{i}=N, \quad \sum_{i=1}^{p(N)} d_{i}^{2}=M_{N},
$$

where:

- $d_{i}$ is the degeneracy order of the $i^{\text {th }}$ eigenvalue of $V_{A}$;

- $M_{N}$ is the number of distinct periodic orbits in $\mathbb{L}_{N} \backslash\{0,0\}$, which coincides with $\operatorname{dim} E_{0}$;

- $E_{0}$ is the invariant eigenspace of $\mathscr{U}_{A}$ of Proposition 2.1;

$-p(N)$ is the common period of the classical closed orbits in $\mathbb{L}_{N} \backslash\{0,0\}$, which coincides with the quantum period, because $N$ is prime [BH, E1, K2].

(2) $W_{A}$, as an operator on $L^{2}\left(\mathbb{T}^{2}, \mu_{N} \times \mu_{N}\right)$, has a point spectrum and each eigenfunction can be written as a sum of functions which are constant on the periodic orbits of rational points with denominator $N$ (cfr. Proposition 2.1). In the classical limit the non-ergodic, zero-entropy, invariant measures $\mu_{N} \times \mu_{N}$ will eventually weakly converge to the Lebesgue measure (with entropy equal to $\log \lambda$, where $\lambda$ is the greatest eigenvalue of $A$ ). This limit measure is ergodic and the Koopman operator has continuous spectrum but for the eigenvalue 1, that is the Wigner functions weakly converge to constant distributions on the torus.

3.3 Eigenvectors and Eigenvalues of the Quantum Propagator. Let us now turn to the construction of a family of eigenstates for $V_{A}$ using the standard facts of analytic number theory recalled in the first part of the Appendix. (To simplify the exposition, we restrict to the case in which we can choose $\theta=(0,0)$ : see Remark (3) after Theorem 3.1.). 
Consider the family of subsets of $\mathbb{L}_{N}$ introduced in Sect. 2.2 (formula (2.11)): to each set $\Lambda_{k, l}$ we associate a "generating function" given by

$$
S_{k, l}(q)=k q^{2}+l q \quad k \in \mathbb{Z}_{N} \quad q, l \in \mathbb{Q}_{N} .
$$

The wave function corresponding to each set $\Lambda_{k, l}$ is naturally obtained by the exponential (in units $\hbar$ ) of its generating function, i.e.:

$$
\psi(q)=c \cdot \exp i \hbar^{-1} S_{k, l}(q)=c \cdot \exp 2 \pi i N S_{k, l}(q)
$$

where $c$ is a normalization constant.

$\forall(k, l) \in \widetilde{\mathbb{Z}}_{N} \times Z_{N}$ and $\forall q \in \mathbb{Z}_{N}$, consider now on $L^{2}\left(S^{1}, \mu_{N}\right) \cong L^{2}\left(\mathbb{Z}_{N}, \mu_{N}\right)$ the set of vectors $\psi_{k, l}(q)$ defined as follows:

$$
\psi_{k, l}(q)=\left\{\begin{array}{l}
\exp \left[\frac{2 i \pi}{N}\left(k q^{2}+l q\right)\right], \quad k \in \mathbb{Z}_{N} \\
(N)^{\frac{1}{2}} \cdot \delta_{l}^{q}, \quad k=\infty .
\end{array}\right.
$$

Using the results concerning Gauss sums listed in Appendix (Formulas (A.6a, b)) we easily obtain the following result (recall once more that, given $x \in \mathbb{Z}_{N}$, it inverse $x^{-1}$ is the unique number in $\mathbb{Z}_{N}$ such that $x x^{-1}=1 \bmod N$ ).

\section{Proposition 3.4.}

(1) For any $k \in \widetilde{\mathbb{Z}}_{N}$

$$
\left\langle\psi_{k, l}, \psi_{k, j}\right\rangle=1 \cdot \delta_{l}^{j}
$$

(2) $\forall k_{1} \neq k_{2}, k_{1}, k_{2} \in \mathbb{Z}_{N}$

$$
\left\langle\psi_{k_{2}, l}, \psi_{k_{1}, j}\right\rangle=\frac{1}{\sqrt{N}} \beta\left(N, k_{1}, k_{2}, l, j\right),
$$

where $|\beta|=1$,

$$
\beta=\left(\frac{\left(k_{1}-k_{2}\right)}{N}\right) \varepsilon_{N} \cdot \exp \left\{-\frac{2 i \pi(l-j)^{2}\left(4\left(k_{1}-k_{2}\right)\right)^{-1}}{N}\right\}
$$

and

$$
\varepsilon_{N}= \begin{cases}1, & N=1 \bmod 4 \\ i, & N=3 \bmod 4\end{cases}
$$

(3)

$$
\left\langle\psi_{k, l}, \psi_{\infty, j}\right\rangle=\frac{1}{\sqrt{N}} \exp \left\{-\frac{2 i \pi}{N}\left[k j^{2}+l j\right]\right\} .
$$

Moreover, the behaviour of these functions under the action of the (representations of the discrete) Heisenberg group is described by the following result: 
Proposition 3.5. $\forall n \in \mathbb{Z}_{N} \times \mathbb{Z}_{N}$,

(1)

$$
\left(\hat{T}(n) \psi_{k, l}\right)(q)=e^{\frac{2 t \pi}{N} \gamma} \cdot \psi_{k, l+n_{1}-2 k n_{2}},
$$

where $\gamma=k n_{2}^{2}-\ln _{2}-2^{-1} n_{1} n_{2}$.

$$
\left(\widehat{T}(n) \psi_{\infty, l}\right)(q)=e^{\frac{2 \pi}{N} n_{1} n_{2}} \cdot e^{\frac{21 \pi}{N} l n_{1}} \psi_{\infty, l+n_{2}} .
$$

Proof. From the construction of the representation we have that for any $\psi$ :

$$
(\hat{T}(n) \psi)(q)=e^{-\frac{2 \pi}{N} n_{1} n_{2}} \cdot e^{\frac{2 i \pi}{N} q n_{1}} \psi\left(q-n_{2}\right),
$$

and the results follow immediately from the definition of the $\psi_{k, l}$.

Remarks.

(1) Note that $\psi_{k, l+n_{1}-2 k n_{2}}$ is the function associated to the set $\Lambda_{k, l+n_{1}-2 k n_{2}}$ which is obtained from $\Lambda_{k, l}$ by a $\left(-\frac{n_{2}}{N}, \frac{n_{1}}{N}\right)$-translation in phase space.

(2) We also have that:

$$
\left\langle\psi_{k, l}, \hat{T}(n) \psi_{k, l}\right\rangle=e^{-\frac{2 \pi}{N} n_{2} l} 1 \cdot \delta_{2 k n_{2}}^{n_{1}},
$$

that is,

$$
\sum_{n \in \mathbb{Z}_{N}^{2}}\left\langle\psi_{k, l}, \hat{T}(n) \psi_{k, l}\right\rangle=N \cdot \delta_{l}^{0}
$$

This is also the value at the origin of the corresponding Wigner function.

This family of functions turns out to be invariant under the action of the quantum propagator. This fact, along with the lattice periodicity, implies that a set of eigenvectors can be constructed by performing linear combinations of such functions. We first have the result:

Lemma 3.3. Let

$$
\zeta: \widetilde{\mathbb{Z}}_{N} \times \mathbb{Z}_{N} \rightarrow \widetilde{\mathbb{Z}}_{N} \times \mathbb{Z}_{N}
$$

be the function uniquely defined by the relation:

$$
A\left(\Lambda_{(k, l)}\right)=\Lambda_{\zeta(k, l)} .
$$

Then

$$
V_{A} \psi_{k, l}=e^{i \sigma(k, l)} \psi_{\zeta(k, l)},
$$

where $\sigma(k, l)$ is a real number for any $k, l$.

Proof. See [DE].

We now recall some basic results concerning the phase space evolution of the subsets $\Lambda_{k, l}$ described in Sect. 2.2. Given $(k, l)$, let $q$ be the period of its orbit under $\zeta$. That is, $\zeta^{s}(k, l) \neq(k, l)$ for $1 \leqq s \leqq q-1$ and $\zeta^{q}(k, l)=(k, l)$. If we denote by $p$ the classical period on the lattice (i.e. the smallest positive integer such that $A^{p}=I_{d}$ $\bmod N$ ), we have seen in Sect. 2.2 that $q=p$ is odd and $q=\frac{p}{2}$ if $p$ is even (i.e. $\left.A^{p / 2}=-I_{d} \bmod N\right)$. 
Now, given any fixed $(k, l)$, set $(\forall m=0, \ldots, q-1)$

$$
\phi_{m}=\frac{c_{N}}{q} \sum_{s=0}^{q-1} e^{i \gamma_{s}} \cdot \psi_{\zeta^{s}(k, l)}
$$

where $\left(\gamma_{0}, \gamma_{1}, \ldots, \gamma_{q-1}\right)$ is recursively defined as:

$$
\gamma_{0}=1, \quad \gamma_{j+1}=\gamma_{j}+\sigma\left(\zeta^{j}(k, l)\right)-\lambda_{m}, \quad j=0,1, \ldots, q-2,
$$

and

$$
\lambda_{m}:=\frac{2 m \pi}{q}+\frac{1}{q} \sum_{j=0}^{q-1} \sigma\left(\zeta^{j}(k, l)\right) .
$$

It is an immediate consequence of the construction that $\phi_{m}$ is an eigenvector with eigenvalue $\lambda_{m}$.

We are now in position to investigate the detailed behaviour of the Wigner functions associated with the above eigenvectors. In particular, we shall give some insight on the actual mechanism which makes the Wigner function a true phase space distribution (namely, positive definite) in the classical limit. To simplify the exposition we will restrict our argument to the case $q=p$ and $l=0$ (even eigenvectors).

Let $W_{k}$ be the Wigner function of the vectors $\psi_{k}: k \in \widetilde{\mathbb{Z}}_{N}$. By Proposition 3.4 and Proposition 3.5 one has, on account of the Gaussian sum (A.6b), supp $W_{k}=\Lambda_{k}$; moreover, $\left.W_{k}\right|_{\Lambda_{k}}=N$. If $k_{0}$ is such that $\Lambda_{k_{0}}$ is an ideal orbit (in the sense recalled in Sect. 2) then obviously $\psi_{k_{0}}$ is an eigenvector by Lemma 3.2 because $A\left(\Lambda_{k_{0}}\right)=\Lambda_{k_{0}}$. Hence the corresponding Wigner function is positive and constant (it is equal to $N$ ) on its support $\Lambda_{k_{0}}$. Note that in this case the weak convergence of the measure $W_{k_{0}} d \mu_{N} \times d \mu_{N}$ to the Lebesgue measure is a consequence of the classical equidistribution of the ideal orbits [BV, PV]. In the general case, $\Lambda_{k_{0}}$ is not invariant under $A$ and different eigenstates are associated to the invariant subsets $\bigcup_{s=0}^{p-1} \Lambda_{k}$ according to formula (3.33a). This represents any eigenfunction $\phi$ as a superposition of the non-orthogonal (by Proposition 3.4) vectors $\psi_{k}: k \in \widetilde{\mathbb{Z}}_{N}$. This non-orthogonality adds interference terms to the constant value $N$ on the Wigner functions. We have indeed (recall that to make the exposition less cumbersome we assume $p=q$ ):

Proposition 3.6. For $m=0, \ldots, q-1$, let $\phi_{m}$ be the eigenvector given by (3.35a), and let $W_{\phi_{m}}$ be its Wigner function. Then:

$$
W_{\phi_{m}}(x)=\frac{\left|\mathcal{c}_{N}\right|^{2}}{q}\left[\sum_{s=0}^{q-1} W_{k_{s}}(x)+\sum_{s \neq t} e^{i\left(\gamma_{t}-\gamma_{s}\right)} W_{k_{s}, k_{t}}(x)\right],
$$

where

$$
W_{k_{s}, k_{t}}(x)=\sum_{n \in \mathbb{Z}_{N}^{2}}\left\langle\psi_{k_{s}}, \hat{T}(n) \psi_{k_{t}}\right\rangle e^{-2 \pi i\langle n, x\rangle} .
$$

Proof. By definition:

$$
W_{\phi_{m}}(x)=\sum_{n \in \mathbb{Z}_{N}^{2}}\left\langle\phi_{m}, \hat{T}(n) \phi_{m}\right\rangle e^{-2 \pi i\langle n, x\rangle} .
$$

Then the results follows by linearity on account of the expression (3.35a). 
Remark. The equidistribution of the Wigner function at the classical limit is thus equivalent to the vanishing of the interference terms, namely the second sum in the r.h.s. of (3.36). These terms are the only ones which may force the Wigner functions to switch sign.

When $N$ is splitting the above construction actually yields a complete orthonormal basis of eigenfunctions as a superposition of orthogonal vectors in such way that, as we shall see in the next section, the interference terms in the Wigner functions vanish as $N \rightarrow \infty$. If $N$ is splitting, we know that the characteristic polynomial splits on $\mathbb{Z}_{N}$; this implies that the function $\zeta(k)-k$ has two zeroes on $\mathbb{Z}_{N}$, i.e. there exists $k_{0} \in \mathbb{Z}_{N}$ such that $\zeta\left( \pm k_{0}\right)= \pm k_{0}$. These values correspond to the linear subspace on the lattice generated by the eigendirections, i.e., the straight lines $\Lambda_{ \pm k}$ of Lemma 2.1(c). For any one of these particular values of $k$, the set $\left\{\psi_{k, l\}}\right\}=0$ gives an orthonormal basis, on which $V_{A}$ acts as a permutation. More precisely:

Proposition 3.7. Let $A$ be of the form (3.11) and let $N$ be splitting with respect to $A$, i.e. let $4 g^{2}-1$ be a quadratic residue of $N$, so that $\pm \sqrt{4 g^{2}-1} \in \mathbb{Z}_{N}$. Then, if $k= \pm \frac{1}{2} \sqrt{4 g^{2}-1}$ one has

$$
V_{A} \psi_{k, l}=\left(\frac{\lambda}{N}\right) e^{-\frac{2 t \pi}{N}(i l)^{2}(g+k)} \psi_{k, i l},
$$

where $\lambda=(2 g+2 k)^{-1} \in \mathbb{Z}_{N}$ is the corresponding eigenvalue of $A$ considered as an endomorphism of $\mathbb{Z}_{N}^{2}$.

Proof. By (3.12), if $A$ is as above the matrix elements $V_{A}\left(q_{1}, q_{2}\right)$ of the propagator have the form

$$
V_{A}\left(q_{1}, q_{2}\right)=\frac{C_{N}}{\sqrt{N}} \exp \frac{2 \pi i}{N}\left(g q_{1}^{2}-q_{1} q_{2}+g q_{2}^{2}\right) .
$$

Therefore, by (3.26) and a straightforward application of the Gaussian sums (A.6a, b) we get:

$$
\begin{aligned}
V_{A} \psi_{k, l}\left(q_{1}\right) & =\frac{C_{N}}{\sqrt{N}} \sum_{q_{2} \in \mathbb{Z}_{N}} \exp \frac{2 \pi i}{N}\left[(g+k) q_{2}^{2}-q_{1} q_{2}+g q_{1}^{2}+l q_{2}\right] \\
& =C_{N} \cdot \varepsilon_{N}\left(\frac{2(g+k)}{N}\right) \exp \left[-\frac{2 \pi i}{N} \frac{l^{2}}{4(g+k)}\right] \psi_{k^{\prime}, l^{\prime}},
\end{aligned}
$$

where

$$
\left\{\begin{array}{l}
k^{\prime}=\frac{4 g k+4 g^{2}-1}{4(g+k)} \\
l^{\prime}=\frac{l}{2(g+k)},
\end{array}\right.
$$

whence the result because the above choice of $k$ yields $k^{\prime}=k$ and the phase factor $C_{N} \cdot \varepsilon_{N}\left(\frac{2}{N}\right)$ can be absorbed in the normalization constant. 
By using this particular basis, we can construct a complete set of eigenfunctions. Denote in what follows by $\langle\lambda\rangle$ the coset of the eigenvalue $\lambda$ of $A$ in $\mathbb{Z}_{N} \times \mathbb{Z}_{N}$, i.e. the subgroup of $\mathbb{Z}_{N}$ cyclically generated by $\lambda$.

Proposition 3.8. Let $V_{A}^{p}=I_{d}$, where $p=\frac{N-1}{m}=\#\langle\lambda\rangle$. Then

(1) $\psi_{k, 0}$ is an eigenvector of $V_{A}$ corresponding to the eigenvalue one.

(2) Write $\mathbb{Z}_{N}=\oplus_{j=0}^{m-1} l_{j}\langle\lambda\rangle,\left(l_{0}=1\right)$; then $\forall l_{j}, j=0, \ldots, m-1$, the family of vectors $\left\{\phi_{j, r}\right\}_{r=0}^{p-1}$ defined as:

$$
\phi_{j, r}=\frac{1}{\sqrt{p}} \sum_{s=0}^{p-1} c_{s} \psi_{k, \lambda^{s} l_{j}}
$$

where $c_{0}=1$ and

$$
c_{s}=\left(\frac{\lambda}{N}\right)^{s} \exp -\left[\frac{2 i \pi s r}{p}\right] \exp -\left[\frac{2 i \pi l^{2}(g+k) \lambda^{2}\left(\lambda^{2 s}-1\right)}{N\left(\lambda^{2}-1\right)}\right] s=1, \ldots, p-1
$$

is such that

$$
V_{A} \phi_{j, r}=e^{-\frac{2 i \pi r}{p}} \phi_{j, r}
$$

$\forall j=0, \ldots, m-1$ and $\forall r=0, \ldots, p-1$.

(3)

$$
\left\langle\phi_{j_{1}, r_{1}}, \phi_{j_{2}, r_{2}}\right\rangle=\delta_{j_{1}}^{j_{2}} \delta_{r_{1}}^{r_{2}} .
$$

Proof. Assertion (1) is (3.38) for $l=0$. To see (2), remark that, once more by (3.38):

$$
\begin{aligned}
V_{A} \phi_{j, r} & =\frac{1}{\sqrt{p}} \sum_{s=0}^{p-1} c_{s}\left(\frac{\lambda}{N}\right) e^{-\frac{2 i \pi}{N}\left(\lambda^{s}+1 l\right)^{2}(g+k)} \psi_{k, \lambda^{s}+1 l} \\
& =\frac{1}{\sqrt{p}} \sum_{s=0}^{p-1} c_{s-1}\left(\frac{\lambda}{N}\right) e^{-\frac{2 i \pi}{N}\left(\lambda^{s} l\right)^{2}(g+k)} \psi_{k, \lambda^{s} l}
\end{aligned}
$$

$\left(c_{-1}=c_{p-1}\right)$. Hence $V_{A} \phi_{j, r}=e^{-\frac{2 \pi n r}{p}} \phi_{j, r}$ iff

$$
e^{-\frac{21 \pi r}{p}} c_{s}=c_{s-1}\left(\frac{\lambda}{N}\right) e^{-\frac{2 i \pi}{N}\left(\lambda^{s} l\right)^{2}(g+k)}
$$

whence (3.39b) because $c_{0}=1$ and $\lambda^{2}\left(1+\lambda^{2}+\cdots+\lambda^{2 s-2}\right)=\frac{\lambda^{2}\left(\lambda^{2 s}-1\right)}{\lambda^{2}-1}$. Finally assertion (3) follows by the unitarity of $V_{A}$ as far as the factor $\delta_{r_{1}}^{r_{2}}$ is concerned, and by (3.27) for the second one.

Remark. This construction yields a complete orthonormal basis of eigenvectors for $\mathscr{H}_{N}$ :

$$
\mathscr{H}_{N}=\psi_{k, 0} \bigoplus_{j=0}^{m-1} \bigoplus_{r=0}^{p-1} \phi_{j, r}
$$

where $\phi_{j, r}: j=0, \ldots, m-1$ are the $m$ distinct eigenvectors corresponding to the $p$ distinct eigenvalues of $V_{A}$ of constant multiplicity $m$. 
Now, given $k$ as before, let us denote by $W_{l_{2}, l_{1}}$ the Wigner function associated to the pair of functions: $\left\{\psi_{k, l_{2}}, \psi_{k, l_{1}}\right\}$ (see Definition 3.2). Then we have:

\section{Proposition 3.9.}

$$
W_{l_{2}, l_{1}}\left(\frac{x_{1}}{N}, \frac{x_{2}}{N}\right)=N \exp -\left[\frac{2 i \pi x_{1}\left(l_{2}-l_{1}\right)}{N}\right] \delta_{x_{2}+2 k x_{1}+\frac{l_{2}+l_{1}}{2}}^{0} .
$$

Proof. By definition

$$
W_{l_{2}, l_{1}}\left(\frac{x_{1}}{N}, \frac{x_{2}}{N}\right)=\sum_{n \in \mathbb{Z}_{N}^{2}}\left\langle\psi_{k, l_{2}}, \hat{T}(n) \psi_{k, l_{1}}\right\rangle e^{-\frac{2 i \pi}{N}\langle n, x\rangle} .
$$

On the other hand, by (3.31a):

$$
\begin{aligned}
W_{l_{2}, l_{1}}\left(\frac{x_{1}}{N}, \frac{x_{2}}{N}\right)= & \sum_{n \in \mathbb{Z}_{N}^{2}} e^{-\frac{2 l \pi}{N}\left(k n_{2}^{2}-2-1_{n_{1}} n_{2}-l_{1} n_{2}\right)} e^{-\frac{2 i \pi}{N}\langle n, x\rangle} \delta_{l_{2}}^{l_{1}+n_{1}-2 k n_{2}} \\
& =e^{-\frac{2 l_{1}}{N} x_{1}\left(l_{2}-l_{1}\right)} \sum_{n \in \mathbb{Z}_{N}} e^{-\frac{2 i \pi}{N} n_{2}\left(x_{2}+2 k x_{1}+2-1_{1}\left(l_{2}+l_{1}\right)\right)} \\
& =e^{-\frac{2 i n x_{1}\left(l_{2}-l_{1}\right)}{N}} N \delta_{x_{2}+2 k x_{1}+\frac{l_{2}+l_{1}}{2}}^{0}
\end{aligned}
$$

Remarks.

(1) If $l_{1}=l_{2}=l, W_{l}\left(\frac{x_{1}}{N}, \frac{x_{2}}{N}\right) \equiv N$ and its support is the line $x_{2}+2 k x_{1}+l=0$, i.e. the ideal line translated by $l$.

(2) If $\phi$ is an eigenfunction of the form constructed in Proposition 3.8,

$$
\phi=\frac{1}{\sqrt{p}} \sum_{s=0}^{p-1} c_{s} \psi_{k, \lambda_{s l}}
$$

(for a given $l$ and $r=0,1, \ldots, p-1$ ) formula (3.36) becomes

$$
W_{\phi}=\frac{1}{p}\left[\sum_{s=0}^{p-1} W_{\lambda, s, \lambda, s l}+\sum_{s \neq t} \bar{c}_{t} c_{s} W_{\lambda, l, i s l}\right] \equiv W_{\phi}^{0}+W_{\phi}^{\text {mix }}
$$

because $\left|c_{s}\right|=1$ for all $s$. Remark that $W_{\phi}^{0} d \mu_{N} \times d \mu_{N}$ is a (normalized, positive) measure taking the constant value $\frac{N}{p}$ on the support $\bigcup_{s=0}^{p-1} \Lambda_{k, \lambda^{s} l}$ which is the union of the translated by $\lambda^{s} l, s=1, \ldots, p-1$ of the ideal line, and therefore, by Lemma 2.1(e), of periodic orbits. Note also that (3.41) differs from (3.26) by the elimination of the $N$ dependence of the overall factor. This allows us to prove the vanishing of $W_{\phi}^{\text {mix }}$ and the (vague) convergence of $W_{\phi}^{0}$ to the constant function 1 as we will see in the next section.

\section{The Classical Limit. Proof of the Main Results}

Having established the quantization prescription $f \rightarrow \hat{f}$ we now study the weak limit points of the matrix elements in the same spirit of the results of $[\mathrm{S}, \mathrm{CdV}, \mathrm{Z}]$ obtained for the Laplacian on compact manifolds where the geodesic flow is 
ergodic or of [HMR] for ergodic flows on compact constant energy surfaces. We emphasize however that in our situation the classical limit for the eigenfunctions is not a priori well defined. The reason is twofold: first, the dimension of the Hilbert space depends on $h=1 / N$ and, second, there is no ordering for the eigenvalues. Hence, in order to define unambiguously the classical limit we introduce the following notations: $\left\{N_{k}\right\}_{k \geqq 1}$ is the sequence of prime numbers greater than a given $N_{0}$ (to be specified later) and set

$$
\mathscr{H}=\bigoplus_{k \geqq 1} L^{2}\left(\mathbb{T}^{1}, d \mu_{N_{h}}\right) .
$$

Given $A \in S L(2, \mathbb{Z})$ set $V_{A}=\left\{V_{A}^{(k)}\right\}_{k \geqq 1}$, where $V_{A}^{(k)}$ acts on $L^{2}\left(\mathbb{T}^{1}, d \mu_{N_{k}}\right)$, as before. In the same way, $\forall \Phi \in \mathscr{H}, \quad \Phi=\left\{\phi^{(k)}\right\}_{k \geqq 1}$, and $\phi^{(k)} \in L^{2}\left(\mathbb{T}^{1}, d \mu_{N_{k}}\right)$, let $W=\left\{W_{\phi^{(k)}}\right\}_{k \geqq 1}$ be the corresponding Wigner function.

\section{Definition 4.1.}

(i) $\Phi \in \mathscr{H}, \Phi=\left\{\phi^{(k)}\right\}_{k \geqq 1}$, is an eigenvector of $V_{A}$ if there exists a sequence $\left\{\lambda^{(k)}\right\}_{k \geqq 1}$, $\hat{\lambda}^{(k)} \in S^{1}$ such that

$$
V_{A}^{(k)} \phi^{(k)}=\lambda^{(k)} \phi^{(k)}, \quad \forall k \geqq 1
$$

(ii) Let $\Phi, \Psi$ be two eigenvectors in $\mathscr{H}$. We say that $\Phi$ and $\Psi$ are distinct, and we write $\Phi \neq \Psi$, if $\phi^{(k)}$ and $\psi^{(k)}$ are distinct eigenfunctions of $V_{A}^{(k)}$ for any $k \geqq 1$. Moreover, we say that $\Phi$ and $\Psi$ are conjugated if, for any $k \geqq 1, \phi^{(k)}$ and $\psi^{(k)}$ belong to the same eigenspace.

Then, to any pair of conjugated eigenvectors $\Phi$ and $\Psi$ we associate a sequence of distributions

$$
(\Phi, \Psi) \rightarrow\left\{d \Omega^{(k)}(\Phi, \Psi)\right\}_{k \geqq 1} \in \mathscr{D}^{\prime}\left(\mathbb{T}^{2}\right)
$$

defined for any smooth functions $f: \mathbb{T}^{2} \rightarrow \mathbb{C}$ by

$$
\int_{\mathbb{T}^{2}} f d \Omega^{(k)}(\Phi, \Psi):=\left\langle\phi^{(k)}, \hat{f} \psi^{(k)}\right\rangle, \quad k \geqq 1 .
$$

The problem is then to find the weak* limit points of the $\left\{d \Omega^{(k)}\right\}$. As already mentioned, the result is formulated in the Banach space $A\left(\mathbb{T}^{2}\right)$, formula (1.7). We have indeed the following

Theorem 4.1. Let $f \in A\left(\mathbb{T}^{2}\right)$, and the sequence $\left\{N_{k}\right\}$ of splitting primes as in Theorem 2.2. Then for any eigenvector $\Phi \in \mathscr{H}$ :

$$
\lim _{k \rightarrow \infty} \int_{\mathbb{T}^{2}} f d \Omega^{(k)}(\Phi, \Phi)=\int_{\mathbb{T}^{2}} f d \mu \equiv f_{0},
$$

and for any pair of conjugated and distinct eigenvectors $\Phi, \Psi \in \mathscr{H}$ :

$$
\lim _{k \rightarrow \infty} \int_{\mathbb{T}^{2}} f d \Omega^{(k)}(\Phi, \Psi)=0
$$

We give two alternative proofs of this theorem. The first one follows from a direct estimate on the matrix elements of the basic observables $\hat{T}(n)$. More precisely: 
Proposition 4.1. Let $g \in \mathbb{N}$ and $N$ be a splitting prime for the map

$$
A=\left(\begin{array}{cc}
2 g & 1 \\
4 g^{2}-1 & 2 g
\end{array}\right) \text {. }
$$

Denote by $\left\{\phi_{j, r}\right\}: r=0, \ldots, p-1$ the set of eigenvectors of Proposition 3.8. Then

(1) if $\left(\phi_{1}, \phi_{2}\right) \in\left\{\phi_{j, r}\right\}_{r=0}^{p-1}$ there is a constant $\Gamma_{1}>0$ (independent on $N$ ) such that, $\forall n \in \mathbb{Z}_{N}^{2}, n \neq(0,0)$,

$$
\left|\left\langle\phi_{2}, \hat{T}(n) \phi_{1}\right\rangle\right| \leqq \frac{\Gamma_{1}}{\sqrt{N}} .
$$

(2) Let $\left\{N_{k}\right\}$ be an increasing sequence of splitting primes such that, for some constant $C>0$,

$$
m_{k}=\frac{N_{k}-1}{p_{k}} \leqq C \quad \forall k \geqq 1
$$

Then there exists $\Gamma_{2}>0$ such that, no matter how two sequences of eigenfunctions $\phi_{j}=\left\{\phi_{j}^{(k)}\right\}_{k \geqq 1}: j=1,2$ are selected, and $\forall n \neq(0,0), n \in \mathbb{Z}_{N}^{2}$ :

$$
\left|\left\langle\phi_{2}^{(k)}, \hat{T}(n) \phi_{1}^{(k)}\right\rangle\right| \leqq \frac{\Gamma_{2}}{\sqrt{N_{k}}} .
$$

Proof. First remark that, by the condition of uniform bounded degeneracy, the second assertion is an immediate consequence of the first one because the elements of all eigenvectors sequences turn out to be finite linear combinations of the vectors $\left\{\phi_{j, r}\right\}_{r=0}^{p-1}$. Moreover to simplify the exposition we can assume without loss of generality $V_{A}^{p}=I_{d}$. Consider first the case in which the eigenvectors $\phi_{1}, \phi_{2}$ correspond to the eigenvalue one. To build arbitrary eigenvectors $\phi_{j}, j=1,2$ corresponding to the eigenvalue 1 we proceed as in Proposition 3.9. Set $(j=1,2)$

$$
\phi_{j}=\frac{1}{\sqrt{p}} \sum_{s=0}^{p-1} c_{s}\left(l_{j}\right) \psi_{\left(k, i, s_{l}\right)}
$$

where $c_{0}\left(l_{j}\right)=1$ and

$$
c_{s}\left(l_{j}\right)=\left(\frac{\lambda}{N}\right) e^{-\frac{2(\pi}{N}\left(i s l_{l}\right)^{2}(g+k)} c_{s-1}\left(l_{j}\right)=\left(\frac{\lambda}{N}\right)^{s} e^{-\frac{2\left(\pi l_{l}^{2}(g+k)^{2}\left(l^{2}-1\right)\right.}{N\left(l^{2}-1\right)}}
$$

for $s=1, \ldots, p-1$. Then

$$
V_{A} \phi_{j}=\phi_{j}, \quad j=1,2 .
$$

By (3.31), (3.32), (3.39d) we can write, using the abbreviations:

$$
\left\{\begin{array}{l}
\theta=k n_{2}^{2}-2^{-1} n_{1} n_{2} \\
\beta=l_{2}^{-1}\left(n_{1}-2 k n_{2}\right) \\
\alpha_{j}=l_{j}^{2}(g+k) \lambda^{2}\left(\lambda^{2}-1\right)^{-1}, \quad j=1,2 \\
\gamma=l_{1} l_{2}^{-1} \\
\alpha_{j}, \beta, \theta, \gamma \in \mathbb{Z}_{N} ;
\end{array}\right.
$$




$$
\begin{aligned}
& \left\langle\phi_{2}, \hat{T}(n) \phi_{1}\right\rangle=\frac{1}{p} \sum_{s, t=0}^{p-1} \bar{c}_{t}\left(l_{2}\right) c_{s}\left(l_{1}\right)\left\langle\psi_{k, \lambda^{t} l_{2}} \hat{T}(n) \psi_{k, \lambda \cdot l_{1}}\right\rangle \\
& =\frac{1}{p} \sum_{s, t=0}^{p-1} e^{\frac{2 \pi t}{N}\left(k n_{2}^{2}-l n_{2} \lambda^{s}-2-1 n_{1} n_{2}\right)}\left\langle\psi_{k, \lambda^{l} l_{2}} \psi_{k, \lambda^{s} l_{1}+n_{1}-2 k n_{2}}\right\rangle \\
& =\frac{e^{\frac{2 \pi t}{N}\left(k n_{2}^{2}-2-1_{1} n_{1}\right)}}{p} \sum_{s, t=0}^{p-1} e^{-\frac{2 \pi i}{N} l_{1} n_{2} \lambda_{s}^{s}} \bar{c}_{t}\left(l_{2}\right) \mathcal{C}_{s}\left(l_{1}\right) \delta_{\lambda^{t} l_{2} l_{2}^{s} l_{1}+n_{1}-2 k n_{2}} \\
& =\frac{e^{\frac{2 \pi i}{N} \theta}}{p} \sum_{s, t=0}^{p-1}\left(\frac{\lambda^{s+t}}{N}\right) e^{-\frac{2 \pi i}{N}\left(l_{1} n_{2}\right) \lambda^{s}} e^{-\frac{2 \pi i}{N}\left[\alpha_{1} \lambda^{2 s}-\alpha_{2} \lambda^{2 t}-\alpha_{1}+\alpha_{2}\right]} \delta_{\lambda^{\prime}}^{\gamma \lambda^{s}+\beta},
\end{aligned}
$$

by Lemma A. 2 we can write (with $\alpha_{3}=l_{1} n_{2}, \alpha_{4}=\theta+\alpha_{1}-\alpha_{2}$ ):

$$
\begin{aligned}
& \left\langle\phi_{2}, \hat{T}(n) \phi_{1}\right\rangle=\frac{e^{\frac{2 \pi t}{N}\left(\alpha_{4}\right)}}{m^{2} p} \sum_{x, y \in \mathbb{Z}_{N^{*}}}\left(\frac{x^{m} y^{m}}{N}\right) e^{\left.-\frac{2 \pi t}{N}\left[\alpha_{3} x^{m}+\alpha_{1} x^{2 m}-\alpha_{2} y^{2 m}\right)\right]} \delta_{y^{m}}^{\gamma x^{m}+\beta} \\
& =\frac{e^{\frac{2 \pi l}{N}\left(\alpha_{4}\right)}}{m^{2} p} \sum_{l=0}^{m-1} \sum_{x, y \in \mathbb{Z}_{N}^{*}}\left(\frac{x^{m} y}{N}\right) \chi_{l}(y) e^{\left.-\frac{2 \pi i}{N}\left[\alpha_{3} x^{m}+\alpha_{1} x^{2 m}-\alpha_{2} y^{2}\right)\right]} \delta_{y}^{\gamma x^{m}+\beta} \\
& =\frac{e^{\frac{2 \pi_{l}}{N}\left(\alpha_{4}\right)}}{m^{2} p} \sum_{l=0}^{m-1} \sum_{x \in \mathbb{Z}_{N^{*}}}\left(\frac{x^{m}\left(\gamma x^{m}+\beta\right)}{N}\right) \chi_{l}\left(\gamma x^{m}+\beta\right) \\
& \times e^{\left.-\frac{2 \pi t}{N}\left[x_{3} x^{m}+\alpha_{1} x^{2 m-}-x_{2}\left(\gamma x^{m}+\beta\right)^{2}\right)\right]},
\end{aligned}
$$

where $\chi_{l}: l=0, \ldots, m-1$ denotes a set of multiplicative characters of order $m$. The result now follows by a straightforward manipulation and a direct application of Theorem A.1.

Let now $\phi_{1}=\phi_{2}=\phi$ be an eigenvector of eigenvalue exp $\left[-\frac{2 \pi i}{p} r\right]: 1 \leqq r \leqq$ $p-1$; then, by $(4.10 \mathrm{a}, \mathrm{b})$ with

$$
c_{s}(l) \equiv c_{s}=e^{-\frac{2 \pi u r}{p}}\left(\frac{\lambda}{N}\right) e^{-\frac{2 l \pi}{N}\left(s^{l} l\right)^{2}(g+k)} c_{s-1}(l),
$$

we have to estimate the sum:

$$
\begin{aligned}
\langle\phi, \hat{T}(n) \phi\rangle & =\frac{1}{p} e^{\frac{2 \pi i}{N} \theta} \sum_{s, t=0}^{p-1} e^{-\frac{2 \pi i}{p} r(s-t)}\left(\frac{\lambda^{s+t}}{N}\right) e^{-\frac{2 \pi i}{N}(\ln 2) \lambda^{s}} e^{-\frac{2 \pi i}{N} \alpha\left[\lambda^{2 s}-\lambda^{2 t}\right]} \delta_{\lambda^{t}}^{\lambda^{s}+\beta} \\
& =\frac{1}{p} e^{\frac{2 \pi i}{N} \theta} \sum_{j, t=0}^{p-1} e^{-\frac{2 \pi t}{p} r j}\left(\frac{\lambda^{j+2 t}}{N}\right) e^{-\frac{2 \pi t}{N}\left(\ln _{2}\right) \lambda^{j+t}} e^{-\frac{2 \pi t}{N} x\left[\lambda^{2}(t+t)-\lambda^{2 t}\right]} \delta_{\lambda^{\prime}}^{\lambda+t+\beta} \\
& =\frac{1}{m^{2} p} e^{\frac{2 \pi i}{N} \theta} \sum_{x, y \in \mathbb{Z}_{N}^{*}} e^{-\frac{2 \pi t}{p} r j(x)}\left(\frac{x^{m} y^{2 m}}{N}\right) e^{-\frac{2 \pi i}{N}\left[\left(l_{2}\right) x^{m} y^{m}+x y^{2 m}\left(x^{2 m}-1\right)\right]} \delta_{y^{m}}^{x^{m} y^{m}+\beta},
\end{aligned}
$$

where $j(x)$ is the unique integer in $\{0, \ldots, p-1\}$ defined by the condition $\lambda^{j(x)}=x^{m}$. Note that the function $\chi(x)=\left(\frac{x^{m}}{N}\right) e^{-\frac{2 \pi i}{p} r(x)}$ is indeed a multiplicative 
character on $\mathbb{Z}_{N}$ of order depending on $m$ and $(r, p)$. Now an easy calculation and Lemma A.2 give an expression of the form

$$
\begin{aligned}
\langle\phi, \hat{T}(n) \phi\rangle= & \frac{1}{m^{2} p} e^{\frac{2 \pi l}{N} \theta} \sum_{l=0}^{m-1} \chi_{l}(\beta) \sum_{x \in \mathbb{Z}_{N}^{*} x^{m} \neq 1} \chi(x) \chi_{l}\left(1-x^{m}\right)^{-1} \\
& \times e^{-\frac{2 \pi l}{N} \beta\left(1-x^{m}\right)-1\left[\left(\ln _{2}\right) x^{m}-\alpha \beta\left(x^{m}+1\right)\right]}
\end{aligned}
$$

$\left(\frac{x^{m}}{N}\right) \chi(x)$ is now a multiplicative character of order depending on $m$ and $(r, p)$. The result is then an immediate consequence of the extension of Theorem A.1 to the rational function of the form $\frac{a x^{m}+b}{1-x^{m}}$ (see [De2, page 190]). The general case $\phi_{1} \neq \phi_{2}$ corresponding to an eigenvalue different from 1 can be easily worked out by combining these two estimates. We omit the details.

Remark. By linearity, the first proof of Theorem 4.1 is an obvious consequence of the estimate (4.9) because $f \in A\left(\mathbb{T}^{2}\right)$.

We turn now to the second proof through determination of the weak* limit of the sequence of distributions (4.3). Let us first state an auxiliary result.

\section{Lemma 4.1.}

$$
d \Omega^{(k)}(\Phi, \Psi)=W\left(\phi^{(k)}, \psi^{(k)}\right) d \mu_{N_{h}} \times d \mu_{N_{h}} .
$$

Proof. Let

$$
f(q, p)=\sum_{n} f_{n} e^{2 \pi i\left(n_{1} q+n_{2} p\right)} .
$$

Then, from Definition (4.4) we have

$$
\int_{\mathbb{T}^{2}} f d \Omega^{(k)}(\Phi, \Psi)=\sum_{n} f_{n}\left\langle\phi^{(k)}, \hat{T}(n) \psi^{(k)}\right\rangle
$$

and, furthermore,

$$
\begin{aligned}
\left\langle\phi^{(k)}, \hat{T}(n) \psi^{(k)}\right\rangle & =\frac{1}{N_{k}^{2}} \sum_{m \in Z_{N_{k}}} e^{\frac{2 \pi i}{N}\langle n, m\rangle} W\left(\phi^{(k)}, \psi^{(k)}\right)(m) \\
& =\int_{\mathbb{T}^{2}} e^{2 \pi i\left(n_{1} q+n_{2} p\right)} W\left(\phi^{(k)}, \psi^{(k)}\right)(q, p) d \mu_{N_{k}}(q) \times d \mu_{N_{h}}(p) .
\end{aligned}
$$

One then obtains the desired result by inserting (4.14) into the r.h.s. of (4.13) and using (4.12).

Now we want to describe the asymptotic behaviour of these distributions. The main fact is that the Wigner functions (associated to a single eigenfunction) not only are constant on each periodic orbit but they actually become constant at the classical limit $N \rightarrow \infty$ also along the "transversal" directions represented by translated ideal lines. Recalling the representation (3.41), we can indeed formalize the assertion of Remark (2) after Proposition 3.9: 
Proposition 4.2. Let $g \in \mathbb{N}$ and $N$ be a splitting prime for the map (4.7). If $\phi \in$ $\left\{\phi_{j, r}\right\}_{r=0}^{p-1}$ then

(1) $\forall n \in \mathbb{Z}_{N}^{2}, n \neq(0,0)$ there is $0<C_{1}(n)<+\infty$, independent of $N$, such that

$$
\begin{gathered}
\int_{\mathbb{T}^{2}} e^{2 \pi i\langle n, x\rangle} W_{\phi}^{0} d \mu_{N} \times d \mu_{N}=0 \quad \text { if } n_{1}-2 k n_{2} \neq 0 \quad \bmod N \\
\left|\int_{\mathbb{T}^{2}} e^{2 \pi i\langle n, x\rangle} W_{\phi}^{0} d \mu_{N} \times d \mu_{N}\right| \leqq \frac{C_{1}}{\sqrt{N}} \text { otherwise } .
\end{gathered}
$$

(2) For any $n \in \mathbb{Z}_{N}^{2}$ there is $C_{2}=C_{2}(n)>0$ such that

$$
\left|\int_{\mathbb{T}^{2}} e^{2 \pi i\langle n, x\rangle} W_{\phi}^{\text {mix }} d \mu_{N} \times d \mu_{N}\right| \leqq \frac{C_{2}}{\sqrt{N}} .
$$

Proof. Concerning the first part we have:

$$
\begin{aligned}
\int_{\mathbb{T}^{2}} e^{2 \pi i\langle n, x\rangle} W_{\phi}^{0} d \mu_{N} \times d \mu_{N} & =\frac{1}{p} \sum_{s=0}^{p-1} \int_{\mathbb{T}^{2}} e^{2 \pi i\langle n, x\rangle} W_{\lambda s l, \lambda^{s} l} d \mu_{N} \times d \mu_{N} \\
& =\frac{1}{N^{2} p} \sum_{s=0}^{p-1} \sum_{x \in \mathbb{Z}_{N}^{2}} N \delta_{x_{2}+2 k x_{1}+\lambda_{s} l}^{0} e^{\frac{2 \pi i}{N}\langle n, x\rangle} \\
& =\frac{1}{N p} \sum_{s=0}^{p-1} e^{-\frac{2 \pi i}{N} n_{2} l l_{s}} \sum_{x_{1} \in \mathbb{Z}_{N}} e^{\frac{2 \pi t}{N} x_{1}\left(n_{1}-2 k n_{2}\right)} \\
& =\frac{1}{p} \sum_{s=0}^{p-1} e^{-\frac{2 \pi t}{N} n_{2} l i^{s}} \delta_{n_{1}}^{2 k n_{2}}
\end{aligned}
$$

where the second equality follows by (3.40), and this proves the first statement. The rest of the proof follows by a direct calculation in the same way as in Proposition 4.1, namely, with $\alpha=l^{2}(g+k) \lambda^{2}\left(\lambda^{2}-1\right)^{-1} \in \mathbb{Z}_{N}$ as above,

$$
\begin{aligned}
& \int_{\mathbb{T}^{2}} e^{2 \pi i\langle n, x\rangle} W_{\phi}^{\text {mix }} d \mu_{N} \times d \mu_{N}=\frac{1}{N^{2} p} \sum_{x \in \mathbb{Z}_{N}^{2}} e^{\frac{2 \pi i}{N}\langle n, x\rangle} W_{\phi}^{\operatorname{mix}}\left(\frac{x_{1}}{N}, \frac{x_{2}}{N}\right) \\
& =\frac{1}{N p} \sum_{x \in \mathbb{Z}_{N}^{2}} \sum_{s \neq t=0}^{p-1}\left(\frac{\lambda^{t+s}}{N}\right) \\
& \times e^{\frac{2 \pi t}{N} x\left(\lambda^{2 t}-\lambda^{2 s}\right)+\frac{2 \pi t}{N} x_{1} l\left(\lambda_{1} s-\lambda^{t}\right)+\frac{2 \pi t}{N}\left(n_{1} x_{1}+n 2 x_{2}\right)} \\
& \times \delta_{x_{2}+2 k x_{1}+\frac{\left(t^{2}+j^{s}\right) t}{2}}^{0} \\
& =\frac{1}{N p} \sum_{s \neq t=0}^{p-1}\left(\frac{\lambda^{t+s}}{N}\right) e^{-\frac{2 \pi t}{N} x\left(i^{2 s}-i^{2 t}\right)} \\
& \times \sum_{x \in \mathbb{Z}_{N}^{2}} e^{-\frac{2 \pi t}{N} x_{1} l\left(\lambda^{t}-\lambda^{s}\right)} e^{\frac{2 \pi t}{N}\left(n_{1} x_{1}-2 k x_{1}-\frac{\left.k^{i}+i^{s}\right)}{2}\right)} \\
& =\frac{1}{p} \sum_{s \neq t=0}^{p-1}\left(\frac{\lambda^{t+s}}{N}\right) e^{-\frac{2 \pi m}{N} \alpha\left(\lambda^{2 s}-\lambda^{2 t}\right)} e^{-\frac{2 \pi t}{N} \frac{\left(i^{t}+i^{s}\right) t}{2}} \\
& \times \delta_{\frac{n_{1}-2 k}{1}}^{\left(\lambda^{t}-\lambda^{s}\right)} \text {. }
\end{aligned}
$$

Theorem A.1 and Lemma A.2 provide the result. 
Remarks.

(1) The extension of this proposition to the Wigner function by an arbitrary pair of eigenvectors of the form (4.10b) can be obtained exactly by the same argument of Proposition 4.1.

(2) The second proof of Theorem 4.1 immediately follows from the above proposition, Formula (3.41) and Formula (4.11).

Let us now interpret these results in the light of the classical equidistribution property proved in Theorem 2.2. According to (4.13), by the unitarity of $\hat{T}(n): n \in$ $\mathbb{Z}^{2}$ we have

$$
\left|\int_{\mathbb{\pi}^{2}} f d \Omega^{(k)}(\Phi, \Psi)\right| \leqq \sum_{n}\left|f_{n}\right|
$$

By this relation and Lemma $4.1 W\left(\phi^{(k)}, \psi^{(k)}\right): k \geqq 1$ may be regarded as a linear functional on $A\left(\mathbb{T}^{2}\right)$ with norm bounded by 1 .

Moreover, by Proposition 3.3(2), if $\phi^{(k)}$ and $\psi^{(k)}$ belong to the same eigenspace, then $W\left(\phi^{(k)}, \psi^{(k)}\right)$ is an eigenfunction with eigenvalue 1 of $\mathscr{U}_{A}^{*}$, the adjoint of the Koopman operator acting on $L^{2}\left(\mathbb{T}^{2}, d \mu_{N_{k}} \times d \mu_{N_{k}}\right)$. In particular, this is true for the Wigner function of any eigenstate $\phi^{(k)}$. Assume obviously $W\left(\phi^{(k)}, \psi^{(k)}\right)$ not constant. In this case, according to Lemma 4.1 and Proposition 2.1, we have the following representation:

$$
d \Omega^{(k)}(\Phi, \Psi)=\sum_{j=0}^{M_{N_{k}}} \alpha_{j}\left(\phi^{(k)}, \psi^{(k)}\right) d \mu_{i,},
$$

where, we recall, $M_{N_{k}}$ denotes the number of closed orbits in $\mathbb{L}_{N_{k}} \backslash\{0,0\}$ (which have one and the same period since $N_{k}$ is prime), and

$$
\mu_{\gamma_{0}}:=\delta_{(0,0)} ; \quad \mu_{\gamma_{1}}:=\frac{1}{p\left(\gamma_{j}\right)} \sum_{k=0}^{p\left(\eta_{1}\right)-1} \delta_{A^{k}(x)}, \quad j=1, \ldots, M_{N_{k}} .
$$

The coefficients $\alpha_{j}: j=0, \ldots, M_{N_{h}}$ may be positive as well as negative; the only condition they have to satisfy is:

$$
\sum_{j=0}^{M_{N_{k}}} \alpha_{j}\left(\phi^{(k)}, \psi^{(k)}\right)= \begin{cases}1, & \text { if } \phi^{(k)} \equiv \psi^{(k)} \\ 0, & \text { otherwise }\end{cases}
$$

which follows from the normalisation condition:

$$
\int_{\mathbb{T}^{2}} W\left(\phi^{(k)}, \psi^{(k)}\right) d \mu_{N_{k}} \times \partial d \mu_{N_{k}}= \begin{cases}1, & \text { if } \phi^{(k)} \equiv \psi^{(k)} \\ 0, & \text { otherwise }\end{cases}
$$

Therefore (4.4), (4.11) and (4.16) yield the representation

$$
\left\langle\phi^{(k)}, \hat{f} \psi^{(k)}\right\rangle=\sum_{j=0}^{M_{N_{h}}} \alpha_{j}\left(\phi^{(k)}, \psi^{(k)}\right) \int_{i j} f .
$$

Moreover, since $W\left(\phi^{(k)}, \psi^{(k)}\right)$ is constant on any single periodic orbit, we have the expression

$$
\alpha_{j}\left(\phi^{(k)}, \psi^{(k)}\right)=\left.\frac{p}{N_{k}^{2}} W\left(\phi^{(k)}, \psi^{(k)}\right)\right|_{i,}
$$


Now (3.41) and (4.19b) yield

$$
\alpha_{j}=\left.\frac{p}{N_{k}^{2}}\left[W_{\phi}^{0}+W_{\phi}^{\mathrm{mix}}\right]\right|_{\gamma} .
$$

On the other hand, $W_{\phi}^{0}$ is a positive constant on its support $\bigcup_{s=0}^{p-1} \Lambda_{s, \lambda s l}$ which is a union of periodic orbits. Since $\left.W_{\phi}^{\text {mix }}\right|_{\eta}$, tends "weakly" to zero, all weights $\alpha_{j}: j=1, \ldots, M_{N}$ in (4.19a) tend in the same sense to be positive and independent of $j$. Hence, by the normalization condition (4.18a), Theorem 4.1 can also be interpreted as a consequence of Theorem 2.2.

Remark. This interpretation clearly shows the intimate connection between the distribution of closed orbits in phase space and the localization properties of the quantum matrix elements. In the analogous case of hyperbolic surfaces, the relation of individual Wigner functions of eigenfunctions to periodic orbits is at best very unclear (see e.g. [Sa]).

We give now some easy consequences of Theorem 4.1 .

Corollary 4.1. Let $\Phi$ be an eigenvector in $\mathscr{H}$ and $W=\left\{W_{\phi^{(k)}}\right\}_{k \geqq 1}$ the corresponding Wigner function. Then

$$
W_{\phi^{(k)}} \longrightarrow 1 \text { for } k \rightarrow \infty
$$

in the weak *-topology of $A\left(\mathbb{T}^{2}\right)$.

Proof. Obvious.

Proof of the Main Theorem. Formulas (1.12) and (1.14) are proved by combining Definition (4.4) and Lemma 4.1. Formula (1.13) follows by the same argument of the proof of Lemma 4.1 on account of the commutation relation.

$$
\left[\hat{f, \hat{g}]}=-\frac{i \hbar}{2 \pi}\{f, g\}^{\wedge}+O(\hbar)\right.
$$

which is an immediate consequence of the commutator of the Heisenberg algebra (3.2):

$$
[\hat{T}(n), \hat{T}(m)]=2 i \sin (\pi \hbar \omega(n, m)) \hat{T}(n+m) .
$$

The uniformity over $n$ is trivial. This proves Assertion (1). Assertion (2) is equivalent to Proposition 4.2, and Assertion (3) is an immediate consequence of Proposition 4.1.

Proof of the Main Corollary. An elementary computation yields

$$
\lim _{m \rightarrow \infty} \frac{1}{m} \sum_{k=0}^{m-1}\left\langle\psi, V_{A}^{k} \hat{f} V_{A}^{-k} \psi\right\rangle=\sum_{n=0}^{N-1}\left|a_{n}\right|^{2}\left\langle e_{n}, \hat{f e_{n}}\right\rangle+\sum_{s \neq r . \lambda_{s}=\lambda_{r}} \bar{a}_{r} a_{s}\left\langle e_{r}, \hat{f e_{s}}\right\rangle
$$

and

$$
\begin{aligned}
\lim _{l \rightarrow \infty} \lim _{m \rightarrow \infty} \frac{1}{m} \sum_{k=0}^{m-1}\left\langle\psi, V_{A}^{k+l} \hat{f} V_{A}^{-l} \hat{g} V_{A}^{-k} \psi\right\rangle= & \lim _{l \rightarrow \infty}\left(\sum_{n=0}^{N-1}\left|a_{n}\right|^{2}\left\langle e_{n}, V_{A}^{l} \hat{f} V_{A}^{-l} \hat{g} e_{n}\right\rangle\right. \\
& \left.+\sum_{s \neq r: \lambda_{s}=\lambda_{l}} \bar{a}_{r} a_{s}\left\langle e_{r}, V_{A}^{l} \hat{f} V_{A}^{-l} \hat{g} e_{s}\right\rangle\right)
\end{aligned}
$$

We obtain the result by combining these relations with the Main Theorem. 


\section{Appendix: Some Basic Results out of Number Theory}

In this section $p$ will denote a prime number and $\mathbb{F}_{q}$ a finite field of characteristic $p$ with $q=p^{r}$ elements (we will be interested mainly in the case $q=p, \mathbb{F}_{q}=\mathbb{Z}_{p}$ ).

Moreover, let $p \in \mathbb{N}$ and $a \in \mathbb{Z}$ be such that $a \neq 0 \bmod p$. Then $a$ is a quadratic residue of $p$ if there is $m \in \mathbb{Z}$ such that $a=m^{2} \bmod p$.

Remarks.

(1) Let $p=N$ be prime, as we shall assume from now on, so that any $x \in \mathbb{Z}_{N}$ has a unique inverse, and consider the second degree equation in the field $\mathbb{Z}_{N}: a x^{2}+b x+c=0 \bmod N,(a, b, c) \in \mathbb{Z}_{N}$. Then, if $b^{2}-4 a c$ is a quadratic residue, $b^{2}-4 a c=m^{2} \bmod N$, the quadratic equation is solvable in $\mathbb{Z}_{N}$ and its roots are $x_{ \pm}=\frac{1}{2 a}(-b \pm m)$.

(2) Likewise, given $A \in \mathrm{GL}(2, \mathbb{Z})$, if $\Delta=4\left(\operatorname{Tr} A^{2}-\operatorname{det} A\right)$ is a quadratic residue, $\Delta=4 D^{2} \bmod N, A$ is diagonable in $\mathbb{Z}_{N}$, with eigenvalues $\lambda_{ \pm}=\frac{1}{2}(\operatorname{Tr} A \pm D)$ and eigenvectors $v_{ \pm}=(1, \pm 2 D)$.

Now, if $\zeta_{p}$ denotes a $p^{\text {th }}$ root of unity, let

$$
\psi:\left(\mathbb{F}_{q},+\right) \longrightarrow \mathbb{C}
$$

be a non-trivial additive $(\psi(x+y)=\psi(x) \psi(y))$ character of $\mathbb{F}_{q}$, and

$$
\chi: \mathbb{F}_{q}^{*} \longrightarrow \mathbb{C}
$$

any (possibly trivial) multiplicative $(\chi(x \cdot y)=\chi(x) \chi(y))$ character. A multiplicative character is of order $m$ if $\chi^{m}$ is equal to the trivial character $\chi_{0}=1$.

Let us give some examples.

Given $N$ prime, let once more $\mathbb{F}_{q}=\mathbb{Z}_{N}$. Then, the only non-trivial multiplicative character of order two is the Legendre symbol $\chi_{2}(x)=\left(\frac{x}{N}\right)$ defined as follows (see e.g. [Ap]):

$$
\left(\frac{x}{N}\right)= \begin{cases}+1 & \text { if } x \text { is a quadratic residue } \\ -1 & \text { otherwise }\end{cases}
$$

Moreover $\left(\frac{0}{N}\right)=0$ for any $x=0 \bmod N$.

The Legendre symbol obviously satisfies the product law

$$
\left(\frac{x y}{N}\right)=\left(\frac{x}{N}\right)\left(\frac{y}{N}\right) .
$$

On the other side, in the present case the group of additive characters is the set

$$
\left\{\psi_{a}(x)=\exp \left\{\frac{2 i \pi}{N} a x\right\} ; \quad a \in \mathbb{Z}_{N}\right\} .
$$

We shall consider generalized sums over finite fields of the type:

$$
\sum_{x \in \mathbb{F}_{\mathcal{G}}} \chi(f(x)) \psi(g(x))
$$


where $\chi$ is a non-trivial multiplicative character of order $d \mid(q-1)$ of $\mathbb{F}_{q}, \psi$ a nontrivial additive character of $\mathbb{F}_{q}$, whereas $f(x), g(x) \in \mathbb{F}_{q}[x]$ are given algebraic functions, for instance polynomials, over $\mathbb{F}_{q}$. We have the

Theorem A.1 (Weil). Let $\chi, \psi$ be a multiplicative character $\neq \chi_{0}$ of order $d$ with $d \mid(q-1)$, and a non-trivial additive character, respectively, of $\mathbb{F}_{q}$. Let $f(x) \in \mathbb{F}_{q}[x]$ admit $m$ distinct roots, and let $g(x) \in \mathbb{F}_{q}[x]$ have degree $n$. Suppose that either

(1) $(d, \operatorname{deg} f)=(n, q)=1$, or, more generally, that

(2) the polynomials $y^{d}-f(x)$ and $z^{q}-z-g(x)$ are absolutely irreducible (i.e irreducible over any finite algebraic extension of $\mathbb{F}_{q}$ ).

Then

$$
\left|\sum_{x \in \mathbb{F}_{q}} \chi(f(x)) \psi(g(x))\right| \leqq(m+n-1) q^{\frac{1}{2}} .
$$

Proof. See, e.g., [Sc], page 45.

Remark that the above result has been obtained by A. Weil as a consequence of the validity of the Riemann conjecture for curves over finite fields [Sc], and an extension to the case where $f, g$ are given rational functions has been achieved by $\mathrm{P}$. Deligne [De1, De2].

Let us briefly discuss some consequences of this result which are used in the paper.

Example A.1. $f(x)=g(x)=x, \chi=\chi_{2}$ and $\psi=\psi_{a}$. Then we have the generalized Gauss sum $G(\psi, \chi)$. If, moreover, $\mathbb{F}_{q}=\mathbb{Z}_{N}$ we obtain the standard quadratic one

$$
g\left(\psi_{a}, \chi_{2}\right)=\sum_{x \in \mathbb{Z}_{N}} \exp \left\{\frac{2 i \pi a}{N} x^{2}\right\}
$$

and, by direct application of Theorem A.1, $\left|g\left(\psi_{a}, \chi_{2}\right)\right| \leqq \sqrt{N}$.

More precise information is contained the following

\section{Proposition A.1.}

$$
g\left(\psi_{a}, \chi_{2}\right)=\varepsilon_{N} \sqrt{N}\left(\frac{a}{N}\right)
$$

where

$$
\varepsilon_{N}= \begin{cases}1, & N=1 \bmod 4 \\ i, & N=3 \bmod 4\end{cases}
$$

and

$$
\sum_{k=0}^{N-1} \exp \left[\frac{2 i \pi}{N}\left(a k^{2}+b k\right)\right]=\varepsilon_{N} N^{\frac{1}{2}}\left(\frac{a}{N}\right) \cdot \exp \left[\frac{-2 i \pi}{N} b^{2}(4 a)^{-1}\right]
$$

if $a \neq 0 \bmod N$,

$$
\sum_{k=0}^{N-1} \exp \left[\frac{2 i \pi}{N}\left(a k^{2}+b k\right)\right]=N \cdot \delta_{b}^{0}
$$

if $a=0 \bmod N$. 
Example A.2. Consider once more the particular case $\mathbb{F}_{q}=\mathbb{Z}_{N}$. Setting $f(x)=x^{2}-4 a b, g(x)=x, \chi=\chi_{2}$ and $\psi=\psi_{1}$ we then find the Kloosterman sum

$$
K l(N, a, b)=\sum_{x \in \mathbb{Z}_{N}}\left(\frac{x^{2}-4 a b}{N}\right) \exp \left\{\frac{2 i \pi}{N} x\right\}=\sum_{x \in \mathbb{Z}_{N}^{*}} \exp \left\{\frac{2 i \pi}{N}\left(a x+b x^{-1}\right)\right\} .
$$

Again, from Theorem A.1, one has the estimate

$$
|K l(N, a, b)| \leqq 2 \sqrt{N} .
$$

The next two results are useful in reducing to the previous case some sums over cyclic subgroups.

Lemma A.1. Suppose $d \mid(q-1)$; then

$$
\sum_{y \text { of order } d} \chi(x)=\left\{\begin{array}{lll}
d & \text { if } & x \in\left(\mathbb{F}_{q}^{*}\right)^{d} \\
0 & \text { if } & x \notin\left(\mathbb{F}_{q}^{*}\right)^{d} x \neq 0 \\
1 & \text { if } & x=0 .
\end{array}\right.
$$

Proof. See [Sc], page 85.

As an immediate consequence, we have:

Lemma A.2. $\forall \lambda \in \mathbb{Z}_{N}^{*}$, denote $\Lambda_{i}=\langle\lambda\rangle$ the cyclic subgroup generated by $\lambda$. Let $\# \Lambda_{\lambda}=\frac{N-1}{m}=p$. If $f: \mathbb{Z}_{N} \times \mathbb{Z}_{N} \longrightarrow \mathbb{C}$ is any complex valued function, then

$$
\sum_{s . t=0}^{p-1} f\left(\lambda^{s}, \lambda^{t}\right)=\frac{1}{m^{2}} \sum_{j, l=0}^{m-1} \sum_{x, y \in \mathbb{Z}_{N}^{*}} \chi_{j}(y) \chi_{l}(x) f(y, x)
$$

where $\left\{\chi_{0}, \ldots, \chi_{m-1}\right\}$ is a set of multiplicative characters of order $m$.

Proof. Clearly $x^{m} \in \Lambda_{i}, \forall x \in \mathbb{Z}_{N}^{*}$, because $\Lambda_{i}$ is exactly the set of roots of the polynomial $x^{p}-1=0$. Moreover, the map $x \longrightarrow x^{m} \in \Lambda_{\text {, }}$, has multiplicity $m$. That is

$$
\sum_{s, t=0}^{p-1} f\left(\lambda^{s}, \lambda^{t}\right)=\frac{1}{m^{2}} \sum_{x, y \in \mathbb{Z}_{N}^{*}} f\left(y^{m}, x^{m}\right)
$$

and the result follows immediately from Lemma A1 because

$$
\sum_{x, y \in \mathbb{Z}_{N}^{*}} f\left(y^{m}, x^{m}\right)=\sum_{j, l=0}^{m-1} \sum_{x, y \in \mathbb{Z}_{N}^{*}} \chi_{j}(y) \chi_{l}(x) f(x, y) .
$$

Using the same technique it is also possible to estimate Kloosterman sums over any cyclic subgroup of $\mathbb{Z}_{N}$, namely

Proposition A.2. $\forall \lambda \in \mathbb{Z}_{N}^{*}$, denote $\Lambda,=\langle\lambda\rangle$ the cyclic subgroup generated by $\Lambda$. Let $\# \Lambda_{;}=\frac{N-1}{m}=p$. Then, $\forall a, b \in \mathbb{Z}_{N}^{*}$,

$$
\left|\sum_{x \in \Lambda_{i}} \exp \left\{\frac{2 i \pi}{N}\left[a x+b x^{-1}\right]\right\}\right|=\left|\sum_{s=0}^{p-1} \exp \left\{\frac{2 i \pi}{N}\left[a \lambda^{s}+b \lambda^{-s}\right]\right\}\right| \leqq C(m) \sqrt{N}(\mathrm{~A} .8)
$$

for some constant $C(m)$ bounded in $m$. 
Proof.

$$
\begin{aligned}
\sum_{s=0}^{p-1} \exp \left\{\frac{2 i \pi}{N}\left[a \lambda^{s}+b \lambda^{-s}\right]\right\} & =\frac{1}{m} \sum_{x \in \mathbb{Z}_{N}} \exp \frac{2 i \pi}{N}\left[a x^{m}+b x^{-m}\right] \\
& =\frac{1}{m} \sum_{j=0}^{m-1} \sum_{x \in \mathbb{Z}_{N}} \chi_{j}(x) \exp \frac{2 i \pi}{N}\left[a x+b x^{-1}\right],
\end{aligned}
$$

where $\chi_{j} j=0, \ldots, m-1$ are the multiplicative characters of order $m$. The assertion now follows from the direct extension of Theorem A.1 to the rational functions of type $a x+b x^{-1}$ (see [De1], [De2], p. 190 and [Sc] p. 85).

Acknowledgements. We are indebted to Jean Bellissard, Italo Guarneri, Anatole Katok and Peter Sarnak for valuable discussions and suggestions.

\section{References}

[AA] Arnold, V.I., Avez, A.: Ergodic Problems in Classical Mechanics. New York: Benjamin, 1968

[Ap] Apostol, T.: Introduction to Analytic Number Theory. Berlin, Heidelberg, New York: Springer, 1976

[BH] Hannay, J.H., Berry, M.V.: Quantization of linear maps on a torus - Fresnel diffraction by a periodic grating. Physica D 1, 267-291 (1980)

[BV] Bartuccelli, F., Vivaldi, F.: Ideal Orbits of Toral Automorphisms. Physica D 39, 194 (1989)

[BNS] Benatti, R., Narnhofer, H., Sewell, G.L.: A non-commutative version of the Arnold cat map. Lett. Math. Phys. 21, 157-172 (1991)

[CdV] Colin de Verdiere, Y.: Ergodicité et functions propres du Laplacien. Commun. Math. Phys. 102, 497-502 (1985)

[Ch] Chandrasekharan, K.: Introduction to Analytic Number Theory. Berlin, Heidelberg, New York: Springer, 1968

[Da] Davenport, H.: On certain exponential sums. Reine, J. Angewandte Mathematik 78, 158-176 (1932)

[DE] Degli Esposti, M.: Quantization of the orientation preserving automorphisms of the torus. Ann. Inst. Poincaré, H. 58, 323-341 (1993)

[De1] Deligne, P.: La conjecture de Weil I. Pub. Math. I.H.E.S. 48, 273-308 (1974)

[De2] Deligne, P.: Cohomologie Etale. Lecture Notes in Mathematics 569, (1977)

[E1] Eckhardt, B.: Exact eigenfunctions for a quantized map. J. Phys. A 19, 1823-1833 (1986)

[E2] Eckhardt, B.: Quantum mechanics of classically non-integrable systems. Phys. Reports 163, 205-297 (1988)

[F] Folland, G.: Harmonic Analysis in Phase Space. Princeton: Princeton University Press, 1988

[FMR] Ford, J., Mantica, G., Ristow, G.H.: The Arnol'd cat: Failure of the correspondence principle. Physica D 25, 105-135 (1991)

[H] Hasse, H.: Number Theory, Berlin, Heidelberg, New York: Springer, 1980

[HMR] Helffer, B., Martinez, A., Robert, D.: Ergodicité et limite semi-classique. Commun. Math. Phys. 09, 313-326 (1987)

[I] Isola, S.: $\zeta$-function and distribution of periodic orbits of toral automorphisms. Europhys. Lett. 11, 517-522 (1990)

[Ka1] Katz, N.: Gauss Sums, Kloosterman Sums, and Monodromy Groups. Princeton, NJ: Princeton University Press, 1988

[Ka2] Katz, N.: Sommes Exponentielles. Asterisque 79, 1980

[Ke1] Keating, J.: Ph. D. thesis, University of Bristol (1989)

[Ke2] Keating, J.: Asymptotic properties of the periodic orbits of the cat map. Nonlinearity 4 , 277-307 (1991) 
[Ke3] Keating, J.: The cat maps: Quantum mechanics and classical motion. Nonlinearity 4, 309-341 (199)

[Kn] Knabe, S.: On the quantization of the Arnold cat map. J. Phys. A 23, 2013-2025 (1990)

[LV] Leboeuf, J., Voros, A.: Chaos revealing multiplicative representation of quantum eigenstates. J. Phys. A 23, 1765-1773 (1990)

[M] Mañe, R.: Ergodic Theory and Differentiable Dynamics. Berlin, Heidelberg, New York: Springer, 1987

[N] Narnhofer, H.: Quantized Arnold cats can be entropic $K$-systems. J. Math. Phys. 33, 1502-1510 (1992)

[PV] Percival I., Vivaldi, F.: Arithmetical properties of strongly chaotic motions. Physica D 25, 105-130 (1987)

[PP] Parry, W., Pollicott, M.: Zeta functions and the periodic orbit structure of hyperbolic dynamics. Astérisque 187-188 (1990)

[RM] Ram Murthy, M.: Artin's conjecture for primitive roots. Mathematical Intelligencer 10, 59-70 (1988)

[S] Schnirelman, A.: Ergodic properties of the eigenfunctions. Usp. Math. Nauk 29, 181$182(1974)$

[Sa] Sarnak, P.: Arithmetic Quantum Chaos. Tel Aviv Lectures 1993 (to appear)

[Sc] Schmidt, W.: Equations over finite fields. An elementary approach. Vol. 536, Lecture Notes in Mathematics, 1976

[VN] Von Neumann, J.: Beweis des Ergodensatzes und des $H$-Theorems in der Neuen Mechanik. Zschr.f.Physik 57, 30-70 (1929)

[Z] Zelditch, S.: Uniform distribution of eigenfunctions on compact hyperbolic surfaces. Duke Math. J. 55, 919-941 (1987)

Communicated by Ya. G. Sinai 
